\title{
Archaeonautica
}

Archaeonautica

L'archéologie maritime et navale de la préhistoire à

l'époque contemporaine

19 | 2017

L'archéologie maritime et navale de la préhistoire à

l'époque contemporaine

\section{À propos de la voile latine : la mosaïque de Kelenderis et les Stereometrica (II, 48-49) d'Héron d'Alexandrie}

Regarding the lateen sail : the Kelenderis mosaic and the Stereometrica (II, 48-49) of Hero of Alexandria

\section{Patrice Pomey}

\section{OpenEdition}

\section{Journals}

Édition électronique

URL : http://journals.openedition.org/archaeonautica/411

DOI : 10.4000/archaeonautica.411

ISSN : 2117-6973

Éditeur

CNRS Éditions

Édition imprimée

Date de publication : 7 décembre 2017

Pagination : $9-25$

ISBN : 978-2-271-11766-3

ISSN : 0154-1854

Référence électronique

Patrice Pomey, «À propos de la voile latine : la mosaïque de Kelenderis et les Stereometrica (II, 48-49) d'Héron d'Alexandrie », Archaeonautica [En ligne], 19 | 2017, mis en ligne le 30 avril 2020, consulté le 10 décembre 2020. URL : http://journals.openedition.org/archaeonautica/411 ; DOI : https://doi.org/ 10.4000/archaeonautica.411 


\title{
À PROPOS DE LA VOILE LATINE: LA MOSAÏQUE de Kelenderis et les Stereometrica (II, 48-49) D'HÉRON D'AlEXANDRIE
}

\author{
Patrice POMEY
}

\begin{abstract}
Résumé
La mosaïque de Kelenderis (Turquie), datée du ve s. ap. J.-C., constitue à ce jour le témoignage le plus précis d'un navire équipé d'une voile de la famille des voiles latines et plus précisément du type dit « voile latine orientale» (settee sail) à voile trapézoïdale. Ce document remonte de ce fait de deux siècles la certitude de l'usage de la voile latine en Méditerranée. Cependant, elle n'a fait l'objet jusqu'à présent que d'interprétations rapides (P. Pomey), voire erronées (Z. Friedmann). Aussi propose-t-on ici une nouvelle étude plus complète et plus détaillée de ce document exceptionnel d'une très grande richesse. Son analyse conduit à admettre que la voile du bateau de Kelenderis est particulièrement élaborée et serait par conséquent le fruit d'un long processus d'évolution. Selon I'hypothèse la plus vraisemblable avancée par certains auteurs (L. Basch), cette origine serait à rechercher du côté d'Alexandrie, foyer réputé d'innovations notamment dans le domaine maritime. Le graffito d'Anfouchi pourrait même faire remonter l'apparition de la pure voile latine triangulaire au $\iota^{\text {er }} \mathrm{s}$. de notre ère, ce qui implique une double évolution conduisant à la voile latine triangulaire et à la voile latine trapézoïdale. Plusieurs indices suggèrent que la communauté Copte aurait joué un rôle important dans cette évolution.
\end{abstract}

L'existence d'une formule de calcul de la surface d'une voile triangulaire du type voile latine chez Héron d'Alexandrie (Stereometrica, II, 48-49), viendrait ainsi confirmer le rôle d'Alexandrie dans le développement de cette voile. Même si sa date et son origine restent incertaines, ce texte s'inscrit dans la tradition Alexandrine et montre I'importance pris par ce nouveau type de gréement.

Le rapprochement de ces deux documents permet d'envisager sous un angle nouveau le problème de l'apparition et de l'évolution de la voile latine.

Mots clefs: Archéologie navale, voile latine, Alexandrie, Antiquité, Méditerranée, mosaïque, Héron d'Alexandrie

Le dossier de la voile latine en Méditerranée n'est toujours pas clos même si des études récentes ont repris la question de son origine et de son évolution (Polzer 2008; Whitewright 2009, 2011). Deux documents méritent à cet égard une attention particulière:

\section{Abstract \\ Regarding the lateen sail: the Kelenderis mosaic and the Stereometrica (II, 48-49) of Hero of Alexandria}

The Kelenderis mosaic (Turkey), dated to the $5^{\text {th }}$ century AD, currently represents the most precise evidence we have of a ship equipped with a sail of the lateen family, and more precisely of the trapezoidal type known as a settee sail. As a consequence, this document demonstrates the certitude of the use of the lateen sail in the Mediterranean two centuries earlier than the previously known evidence. However, it has until now only been the subject of brief and even mistaken interpretations (by P. Pomey and Z. Friedmann respectively). Here we propose a new, more complete and detailed study of this exceptional document of great interest.

The analysis leads to the admission that the sail on the Kelenderis boat is particularly elaborate and consequently would have been the result of a long process of evolution. According to the most probable hypothesis put forward by certain authors (L. Basch), the origins should be looked for in Alexandria, a reputed home of innovations, especially in the maritime domain. The graffito of Anfushi could even date the appearance of the pure triangular lateen sail back to the $1^{\text {st }}$ century AD, which would imply a double development leading to the triangular lateen sail and to the trapezoidal lateen sail. Several clues suggest that the Coptic community may have played an important role in this evolution.

The existence of a formula for calculating the surface area of a triangular sail of the lateen type in the work of Hero of Alexandria (Stereometrica, II, 48-49) would seem to confirm the role of Alexandria in the development of this sail. Even if its date and origin remain uncertain, this text sits within the Alexandrian tradition and shows the importance given to this new type of rigging.

By examining these two documents side by side, one can approach the question of the appearance and development of the lateen sail from a new angle.

Key words: naval archaeology, lateen sail, Alexandria, antiquity, Mediterranean, mosaic, Hero of Alexandria l'un, la mosaïque navale de Kelenderis, doit être reconsidéré en raison de son originalité et de son intérêt; l'autre, un passage des Stereometrica (II, 48-49) d'Héron d'Alexandrie, jusqu'à présent totalement ignoré, doit être versé au dossier. 


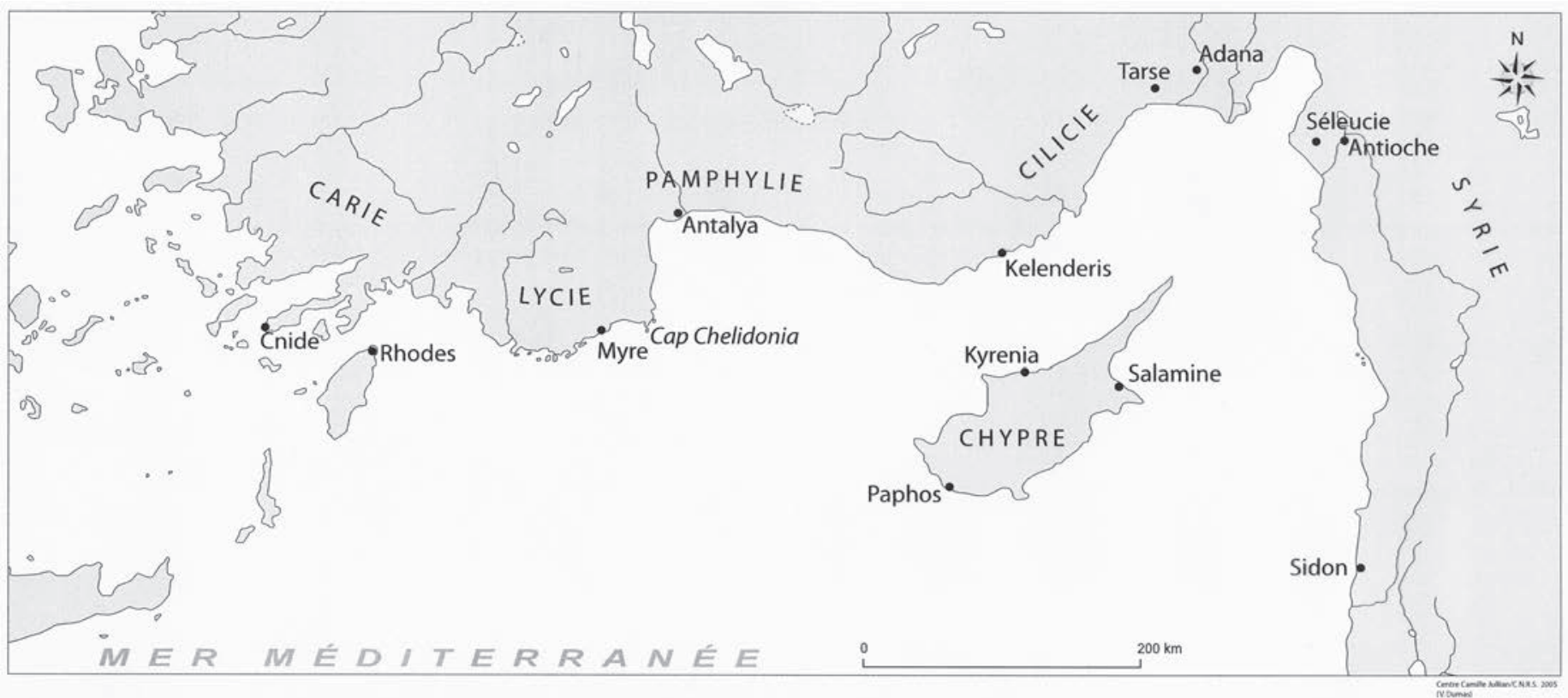

Fig. 1: Côte sud de la Turquie avec la situation de Kelenderis en Cilicie (Dessin V. Dumas, Aix Marseille Univ, CNRS, Minist Culture \& Com, CCJ, Aix-en-Provence, France).

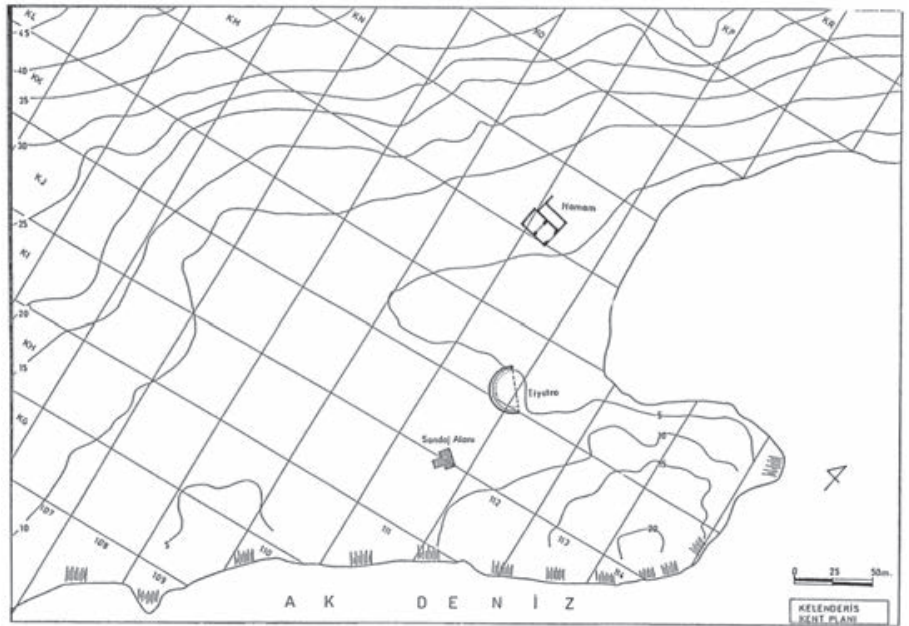

Fig. 2: Plan général du site de Kelenderis avec le site des fouilles (sondaj Alanı) et l'anse du port (Zoroglu 1992).

\section{LA MOSAÏQUE NAVALE DE KELENDERIS}

Découverte en 1992, dans l'ancien port de Kelenderis, aujourd'hui Aydincik (Turquie), sur la côte de Cilicie $^{1}$ (fig. 1, 2, 3), la grande mosaïque navale offre un témoignage exceptionnel sur le développement de la voile latine antique. Ce document constitue en effet, à ce jour, le plus ancien exemple d'un gréement appartenant à la famille des voiles latines dont l'interprétation et la datation font le plus consensus ${ }^{2}$. Outre son intérêt du point de vue de l'histoire

1. Zoroglu 1992, 1993, 1994, 1996; Gates 1994, 1995. Je tiens à remercier le Prof. L. Zoroglu de son obligeance et pour tous les renseignements qu'il a bien voulu me communiquer à l'époque au sujet de cette mosaïque. Je tiens aussi à remercier tout particulièrement Mme Véronique Blanc-Bijon, Ingénieur de Recherche au Centre Camille Jullian (Aix Marseille Univ, CNRS, Minist Culture \& Com, CCJ, Aix-en-Provence, France), spécialiste des mosaïques, pour m'avoir signalé ce document dès 1998 et pour toute l'aide qu'elle m'a apportée dans cette étude.

2. La partie consacrée dans le présent article au navire de la mosaïque de Kelenderis

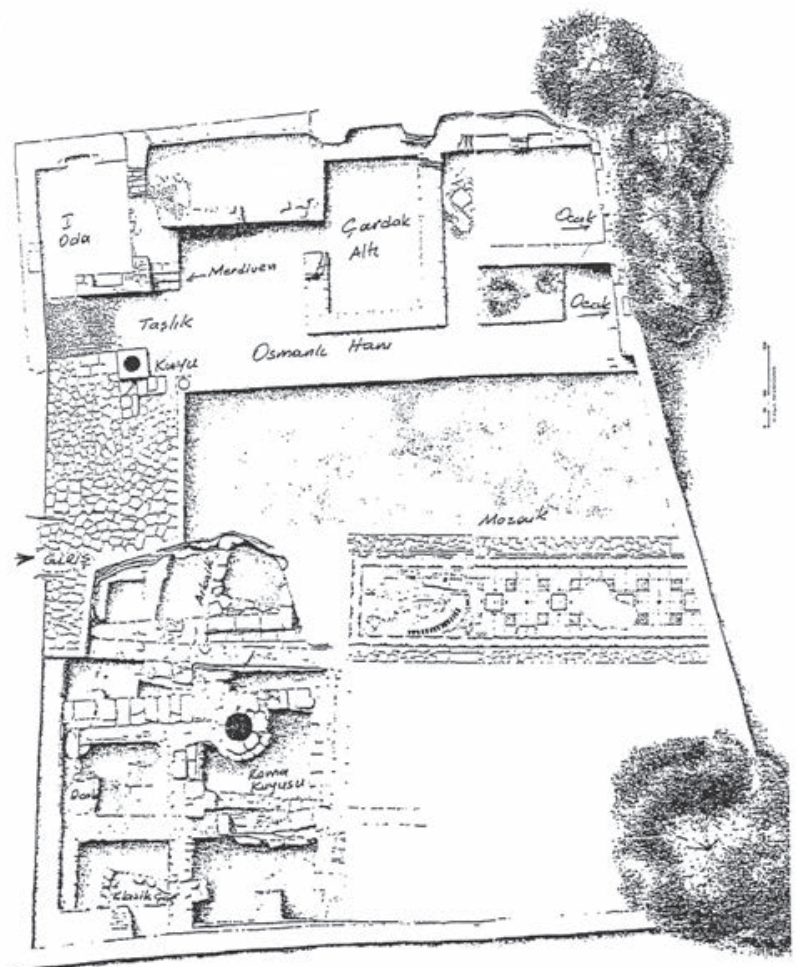

Fig. 3: Plan des fouilles de l'édifice à mosaïque (Zoroglu 1994).

reprend l'essentiel de ma communication au $9^{\text {th }}$ International Symposium on Ship Construction in Antiquity intitulée "Un nouveau témoignage sur la voile latine: la mosaïque de Kelenderis (v. 500 ap. J.-C.; Turquie)", tenu à Agia Napa (Chypre), fin août 2005. Cette dernière a donné lieu à un article préliminaire "The Kelenderis Ship: A Lateen Sail" (Pomey 2006) en réponse à l'article de Zaraza Friedman et Levent Zoroglu (2006), "Kelenderis Ship - Square or Lateen Sail ?", dans lequel les auteurs interprétaient à tort la voile du navire comme une voile carrée traditionnelle, hypothèse déjà avancée par Z. Friedman (2003). L'interprétation du gréement du navire de Kelenderis comme appartenant à la famille des voiles latines a été confirmée à l'époque par Owain T. P. Roberts (2006) et Lionel Casson (2006), contestant à leur tour l'interprétation erronée de Z. Friedman et L. Zoroglu, et a été depuis suivie par tous les auteurs (p. ex. Polzer 2008; Whitewright 2009, 2011). Néanmoins, Z. Friedman (2007) persiste contre toute évidence dans son interprétation fautive. 


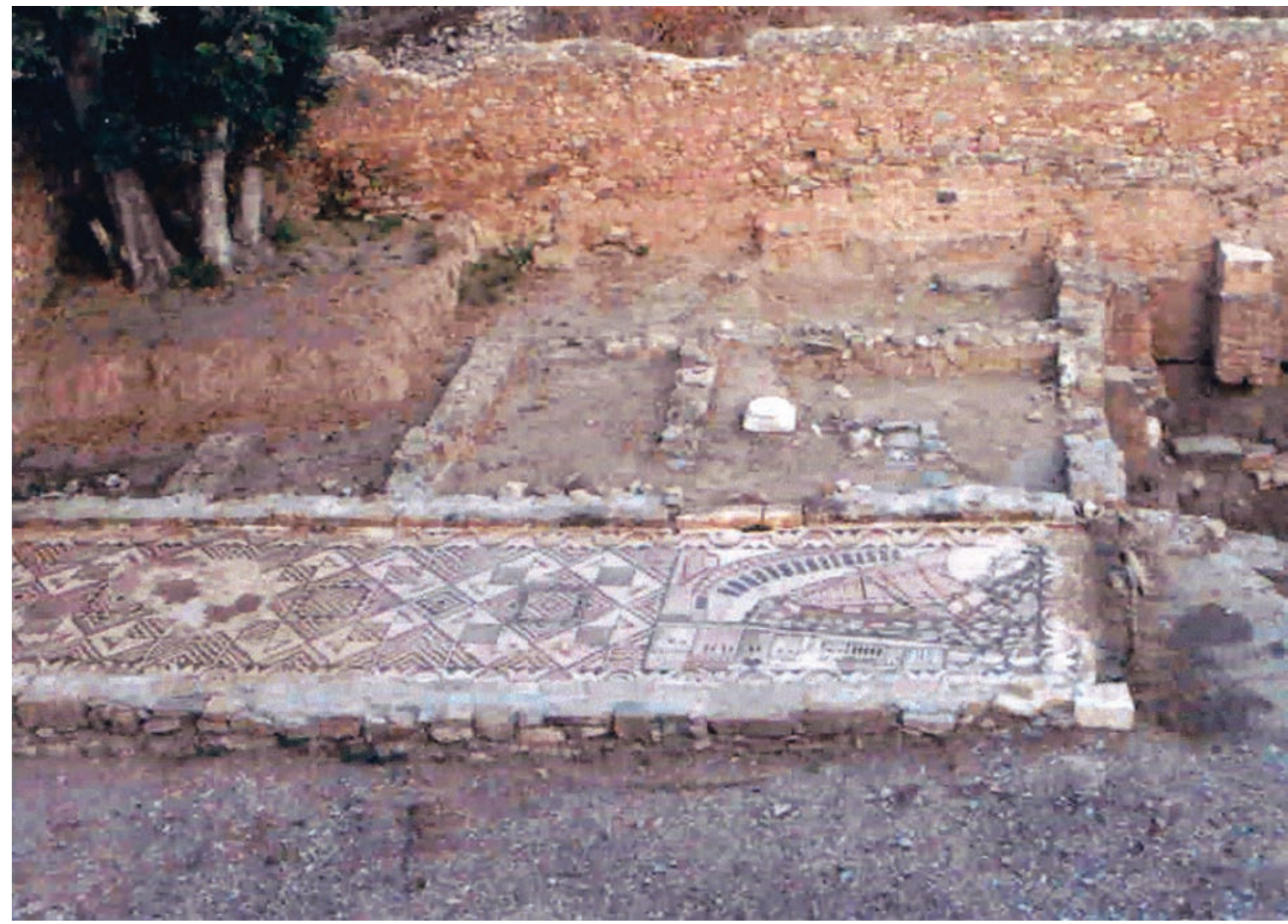

Fig. 4: Vue d'ensemble de la mosaïque (Photo Kelenderis Projesi).

navale par la précision de ses détails, cette mosaïque est aussi, et avant tout, un remarquable document décoratif qui obéit à des règles de représentation dont la compréhension est indispensable à son interprétation. De ce fait, son analyse mérite d'être reprise et approfondie.

La mosaïque se présente sous la forme d'un long tapis décoratif polychrome de 3,20 m de large qui a été dégagé sur $12 \mathrm{~m}$ de longueur sans que ses limites soient pour autant atteintes. Elle se compose, pour l'essentiel, sur $9 \mathrm{~m}$ de longueur, d'un décor géométrique qui s'achève par un panneau figuratif de $3 \mathrm{~m}$ sur $3 \mathrm{~m}$ représentant une scène portuaire (fig. 4). L'ensemble est entouré d'une frise continue de fleurs de lotus tête-bêche. Le pavement devait orner un grand bâtiment dont la fonction reste indéterminée (thermes?). Selon les données stratigraphiques, la mosaïque est postérieure aux niveaux des $\operatorname{III}^{\mathrm{e}}$ et $\mathrm{IV}^{\mathrm{e}}$ siècles sur lesquels elle repose et le Prof. Zoroglu propose, en tenant compte des critères stylistiques, de la dater avec précaution vers 500 ap. J.-C., voire dans la deuxième moitié du $\mathrm{V}^{\mathrm{e}}$ siècle, sans pour autant totalement exclure une date plus récente (Zoroglu 1992, 1993, 1994, 1996). Cependant, selon certains spécialistes, l'appartenance de la mosaïque au ve siècle est assurée et sa datation remonterait très certainement à la première moitié de ce siècle ${ }^{3}$.

3. Cette datation repose sur la trame géométrique de l'ensemble et notamment sur le quadrillage de bandes déterminant des carrés d'entrecroisement et de grandes cases, à décor en arc-en-ciel, cf. Balty 1977, 1995; Donceel-Voûte 1988, 1994. Mmes Pauline Donceel-Voûte et Janine Balty ont bien voulu me faire part de leurs
La scène qui nous intéresse représente un navire sous voile qui entre dans un port en traînant en remorque deux embarcations plus petites (fig. 5). Si dans l'ensemble le panneau de la scène navale est en bon état et ne semble pas avoir fait l'objet de restauration, on note cependant que la partie arrière du navire principal, en avant du gouvernail latéral tribord, est altérée.

Le port, identifié par le Prof. L. Zoroglu comme étant le port antique de Kelenderis, est figuré selon un schéma classique évoquant la forme d'un hémicycle ouvert sur la droite. Il comporte, dans sa partie inférieure un môle droit s'avançant vers la mer où figure une suite d'une dizaine de constructions diverses parmi lesquelles L. Zoroglu identifie: le mur d'enceinte de la ville, une porte, un entrepôt, une tour, une taverne en avant des murailles et, en arrière, une église. Ce môle et ces bâtiments sont représentés d'une façon originale comme s'ils étaient vus depuis le rivage opposé, c'est-à-dire à l'envers, tête en bas, par rapport au point de vue principal de la mosaïque donné par le grand navire. Les autres côtés du port, représentés cette fois à l'endroit, sont constitués par un rivage curviligne longé par un long bâtiment à portique en arcades qui pourrait être, selon L. Zoroglu, un grand entrepôt, en arrière duquel figure un édifice isolé à trois voûtes qu'il interprète comme étant des thermes. L'ensemble est figuré dans des tons beige-rosés tirant parfois vers le marron. Les contours de la plupart des bâtiments sont soulignés d'un trait noir

avis éclairés de spécialistes des mosaïques orientales pour la datation de cette mosaïque. Qu'elles en soient très sincèrement remerciées. 


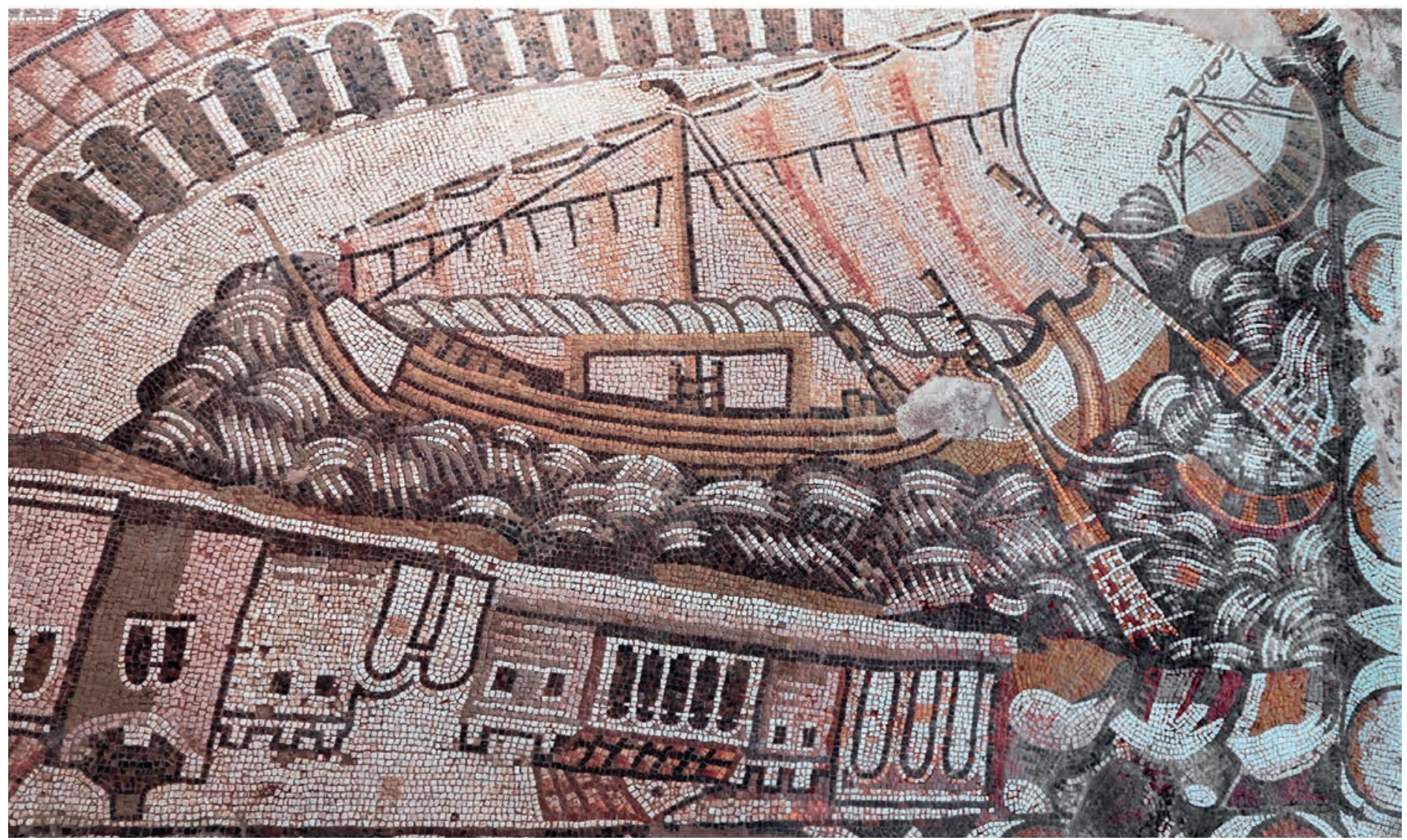

Fig. 5: Vue de détail du panneau de la mosaïque avec la scène nautique (Photo @ birbakistamersin.org - Ahmet Gullu).

et les ouvertures sont généralement foncées, sauf dans deux exceptions où elles sont claires.

Entrant dans le bassin portuaire aux flots agités et se dirigeant donc vers la gauche, s'avance un grand navire sous voile naviguant bâbord amure. Il traîne en remorque deux embarcations annexes (epholkia ou scaphae): à bâbord, une simple barque à deux bancs; à tribord, une autre barque, de type semblable, mais plus grande avec quatre bancs et qui est remorquée sous voile. Ce mode de représentation associant remorque et voile peut paraître paradoxal mais est bien attesté par ailleurs, notamment par le graffito du navire Europa à Pompéi et, pour une époque plus proche de celle qui nous intéresse, par les bateaux de la fresque d'Eboda $\left(\mathrm{VI}^{\mathrm{e}} \mathrm{s} \text {. ? }\right)^{4}$. En revanche, la représentation de deux barques en remorque est à ma connaissance originale même si l'existence de plusieurs annexes pour des navires importants est bien attestée par les textes ${ }^{5}$.

La scène se veut réaliste et démonstrative. Elle est démonstrative par la multiplicité des points de vue adoptés par le mosaïste: double, comme on l'a souligné, pour le port; quadruple pour les bateaux. En effet, si les deux embarcations en remorque sont vues de dessus et de trois quarts, le grand

4. Le graffito du navire Europa a été maintes et maintes fois représenté, cf. notamment Maiuri 1958, p. 21, fig. 2; Basch 1987, p. 469, fig 1051; Pomey 1997b, p. 83 ; pour la fresque d'Eboda, cf. Basch 2001, p. 70-71 et fig. 19. Voir aussi le sarcophage de Cornelius Arrianus ( $\mathrm{I}^{\mathrm{er}}-\mathrm{II}^{\mathrm{e}} \mathrm{S}$. ap. J.-C.) au musée de Sinope (Turquie) où un grand navire à deux mâts avec voiles carrées et suparum traîne en remorque une barque sous voile.

5. Sur les barques annexes, cf. Casson 1971, p. 248-249, n. 93-97; Pomey 1997b, p. $12-17,83-84$ navire est vu à la fois de côté pour l'essentiel de la coque, de trois quart avant pour la proue et de trois quarts arrière pour la poupe. Elle est réaliste comme le montre le souci du mosaïste de rendre l'agitation des flots en évoquant l'écume blanche à la crête des vagues et de représenter les bateaux «dans leurs lignes » et non posés, plus ou moins artificiellement, sur la mer comme cela arrive souvent dans l'iconographie antique. De ce fait, les coques des deux barques en remorque sont en grande partie masquées par les flots et sont à peine visibles. De même pour le grand navire, dont la coque n'est figurée que jusqu'à la ligne de flottaison alors que les œuvres vives de la carène et la quille disparaissent sous les eaux. Notons aussi l'effet de vue en transparence de la partie inférieure immergée des pelles des deux gouvernails latéraux et le rendu des filets d'eau laissés par le sillage des safrans. De tels modes de représentation sont assez rares pour mériter d'être soulignés ${ }^{6}$.

\section{LE NAVIRE}

Le navire principal, totalement hors d'échelle par rapport au décor, occupe à lui seul tout l'espace du bassin portuaire et constitue manifestement, avec le port, le sujet principal de la mosaïque. Le fait qu'il traîne en remorque deux embarcations annexes dont l'une

6. Parmi quelques parallèles possibles de navire représentés "dans leurs lignes" au milieu de flots agités, on notera le relief de Portus de la collection Torlonia (Casson 1971, fig. 144; Basch 1987, fig. 1038; Pomey 1997b, p. 82) et, mieux encore, la scène du sarcophage d'Ostie de la Ny-Carlsberg Glyptothek de Copenhague (Casson 1971, fig. 147; Basch 1987, fig. 1038; Pomey 1997 b, p. 85). 


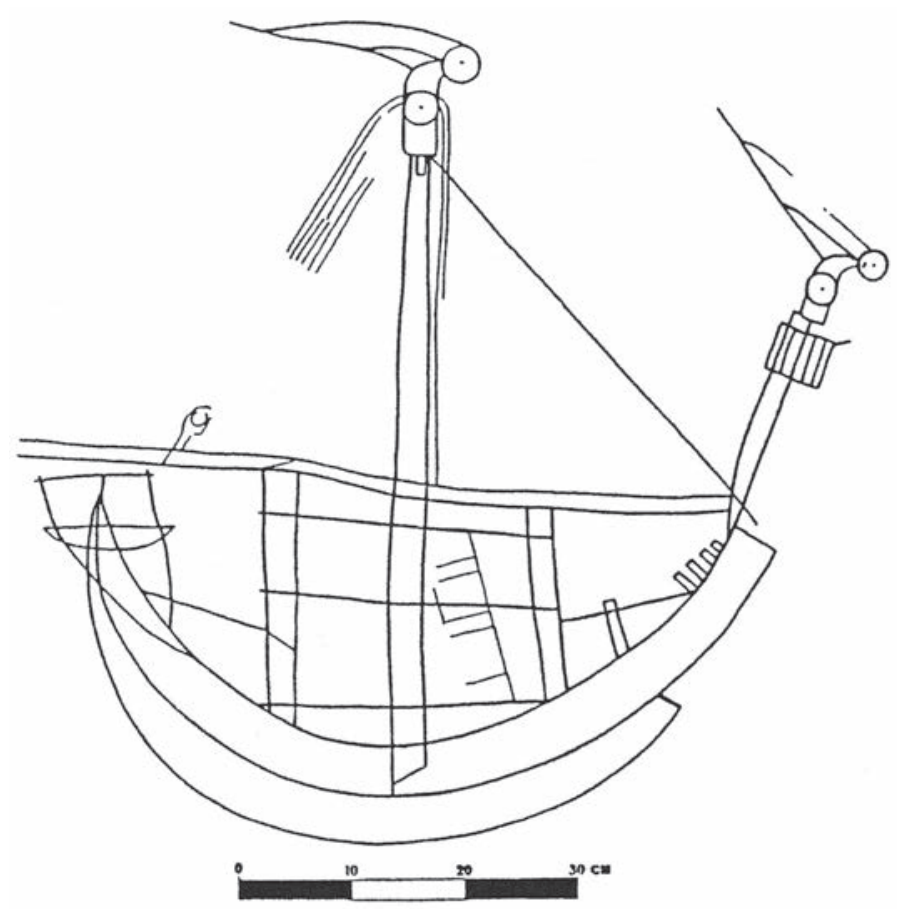

Fig. 6: Graffito de navire marchand byzantin, Corinthe (ve ou ve siècle.) (Basch 1991a).

est voilée indique un navire d'une certaine importance et tout laisse penser qu'il s'agit vraisemblablement d'un bateau de commerce. Comme nous venons de le voir, la coque est représentée selon un triple point de vue où à la vision latérale du flanc s'ajoutent les vues trois quarts avant et arrière des extrémités. Cette distorsion visuelle confère à la poupe une forme étrange où l'on semble discerner trois étambots ! La poupe est en fait manifestement déformée par suite d'une erreur d'interprétation du mosaïste qui a éprouvé des difficultés pour raccorder la vue perspective de l'arrière à la vue latérale du flanc de la coque. Aussi, on interprétera la poupe comme une poupe traditionnelle à étambot axial convexe encadré, non par deux autres étambots, mais par les extrémités verticales proéminentes des ailes de protection des gouvernails latéraux. Dans un effet de perspective mal compris, ce sont les extrémités des ailes latérales que le mosaïste a fait figurer comme intégrées à la courbure de la coque. La position des gouvernails latéraux et la représentation de la caisse de l'aile sur le flanc bâbord du navire ne laissent aucun doute à ce sujet. La proue est, de même, déformée par la vision trois quart avant qui répond à la vision trois quarts arrière de la poupe. De ce fait, sous l'étrave proéminente apparaît une partie du flanc tribord qui serait normalement invisible dans une vision latérale. L'étrave, elle aussi convexe, se relève cependant selon une courbure moins prononcée et plus ouverte que celle de l'étambot qui confère à l'avant du navire un élancement plus important qu'à l'arrière. Ce profil de coque - qui relève du type général dit «symétrique» selon la terminologie de P.-M. Duval (Duval 1949) - est bien attesté dans l'iconographie navale antique ${ }^{7}$ bien qu'il soit moins fréquent que le profil inverse, caractérisé par un élancement de l'étambot plus important que celui de l'étrave.

Notons aussi que la proue et la poupe sont figurées en blanc, contrairement au reste de la coque de couleur «bois», marron clair, ce qui suggère la présence d'une peinture de coque. Les contours des différents éléments de la coque sont soulignés d'un trait noir.

7. Cf. par exemple Casson 1971, fig. 142, 149; Basch 1997, fig. 1048, 1049.

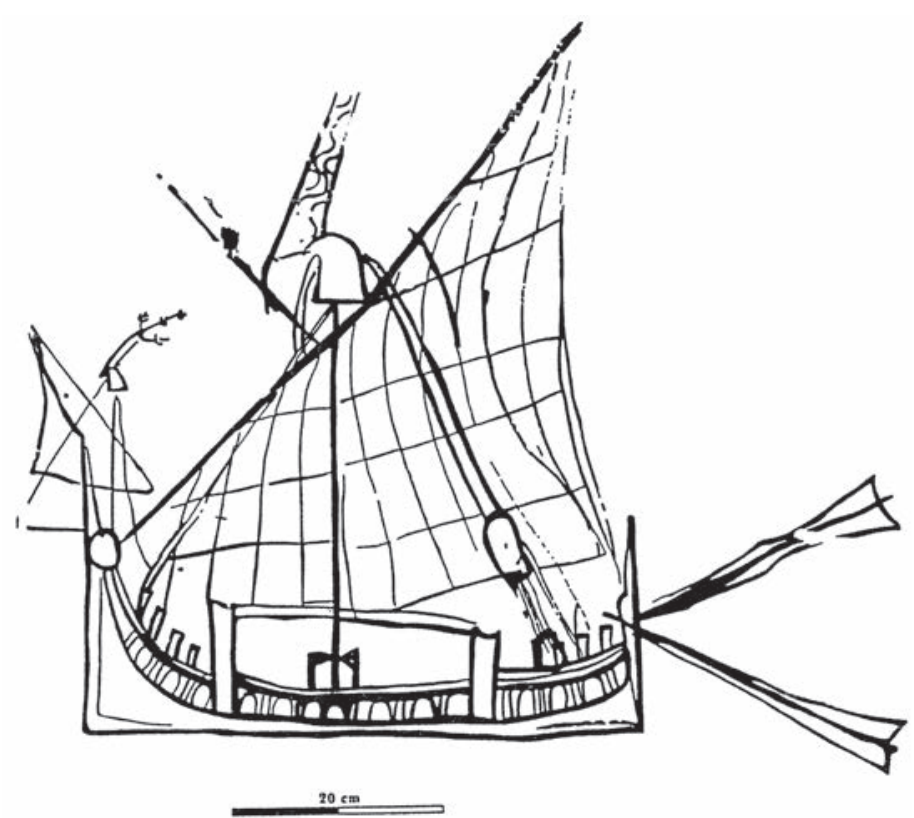

Fig. 7: La felouque des Kellia (VII' siècle.) (Basch 1991b).

Le long du flanc bâbord, on note la caisse latérale de l'aile de protection du gouvernail qui prend naissance sur la proue, en arrière de l'étrave, et qui se prolonge le long de la coque jusqu'à la poupe où elle semble s'arrêter juste en avant de l'étambot. Elle est composée de trois rangées de bordages, nettement individualisés par des lignes noires, et elle s'achève par un montant vertical proéminent - en partie altéré sur la mosaïque - source de l'erreur de perspective que nous avons soulignée. La représentation de telles ailes latérales de protection du gouvernail, selon cette même structure, est bien attestée dans l'iconographie navale romaine qui en présente de nombreux exemples ${ }^{8}$.

Sur le plat-bord, on distingue ce qui semble être très probablement des bittes d'amarrage: cinq sur l'avant et au moins deux sur l'arrière compte tenu de la lacune de la mosaïque. Aucun indice ne permet, en revanche, de dire si le bateau est ponté ou non. Cependant au centre de la coque, entourant le mât principal, figure une grande structure rectangulaire dont le contour est de couleur marron souligné intérieurement d'un filet noir. Cette structure laisse apercevoir le bas du mât et ne peut donc pas être une cabine fermée. Il est tout aussi peu probable qu'il s'agisse d'une structure destinée à recevoir une cargaison en pontée comme on en connaît plusieurs exemples. Dans ce dernier cas, la structure est plus complexe et est généralement compartimentée en plusieurs niveaux (Pomey 1993). En revanche, cette structure se retrouve d'une façon identique sur un graffito de navire byzantin de Corinthe ( $\mathrm{v}^{\mathrm{e}}$ ou $\mathrm{VI}^{\mathrm{e}} \mathrm{s}$.) (fig. 6) et sur le dipinto de la felouque des Kellia en Égypte (VII ${ }^{\mathrm{e}} \mathrm{s}$.) (fig. 7). Elle a été clairement identifiée par L. Basch comme une plate-forme où devait prendre place des combattants, le xylokastron, caractéristique des bateaux byzantins (Basch 1991a, 1991b). Cette plateforme de combat, comme le montrent le navire de la mosaïque de Kelenderis et le bateau de Corinthe, n'était pas l'apanage exclusif des navires de guerre mais pouvait aussi équiper, à des fins défensives, les navires marchands.

8. Cf. par exemple, Casson 1971, fig. 142, 144, 147, 149; Basch 1997. 
L'appareil de gouverne, constitué de deux grandes rames gouvernails latérales, frappe par sa taille et sa simplicité. En dimensions, les rames gouvernails apparaissent plus grandes que le mât principal et sont pratiquement équivalentes aux deux tiers de la longueur du navire, ce qui est considérable. Le dispositif est en outre d'une grande simplicité. Chaque gouvernail est apparemment constitué d'une simple rame, dépourvue de barre de manœuvre (clavus), dont la mèche axiale se prolonge jusqu'en bas de la pelle. Cette dernière représente en proportion les 2/5 de l'ensemble. Son extrémité inférieure est échancrée en forme de trident dont les pointes sont constituées par les extrémités de la mèche et des bords d'attaque et de fuite du safran. En fait, il est probable que l'ensemble n'était pas monoxyle, comme le serait une véritable rame, et que les parties avant et arrière de la pelle venaient s'assembler sur la mèche. On interprétera, en effet, la petite bande blanche qui traverse chacun des safrans dans sa partie supérieure, au-dessus du niveau de l'eau matérialisé par les filets d'eau du sillage, comme une pièce de renfort (ferrure?) de l'assemblage mèche-safran. Chaque gouvernail repose à l'intérieur de l'extrémité de chaque aile en un seul point situé sensiblement au milieu de la mèche ${ }^{9}$. En revanche, la position très en arrière et très libre des deux rames gouvernails et leur grande dimension, qui évoquent la façon d'agir d'un aviron de queue, est originale. On retrouve, cependant, ces mêmes particularités, y compris dans la forme à trois pointes de la base du safran, dans l'appareil de gouverne du navire des Kellia et dans l'iconographie byzantine (Basch 1991a, fig. 3A, 3B; 1991b).

\section{LE GRÉEMENT DU NAVIRE}

Le gréement latin reste la partie la plus remarquable de ce bateau déjà étonnant à bien des égards. Le mât principal, l'arbre de mestre selon le vocabulaire méditerranéen ${ }^{10}$, est situé en position centrale et est très légèrement incliné sur l'avant. Sa partie inférieure est maintenue à l'aide de deux étais verticaux, situés en avant et en arrière de l'arbre, les chelamides, auxquels le mât est très clairement ligaturé ${ }^{11}$. Ce même dispositif se retrouve sur la felouque des Kellia. À son extrémité supérieure, le mât s'achève par un motif de couleur noire en forme de crosse dont la pointe, tournée vers l'avant, s'achève par un petit motif circulaire de couleur ocre-rouge. On identifiera à coup sûr ce motif comme étant un calcet destiné à recevoir, en tête de mât, les poulies du gréement courant de la voilure. L'une de ces poulies, située à l'extrémité de la pointe de la crosse, serait représentée par le petit motif circulaire de couleur ocre-rouge. Ce type de calcet à la forme très particulière est caractéristique des gréements byzantins comme l'a souligné L. Basch (1991a, 1991b). On le retrouve en effet sur le navire du graffito de Corinthe, où il figure avec deux poulies dont l'une est fixée à l'extrémité de la pointe de la crosse, à nouveau sur le navire des Kellia et sur d'autres exemples byzantins. Le mât est maintenu sur l'avant par un étai fixé juste en arrière de l'étrave ${ }^{12}$.

9. La fixation du gouvernail latéral entre la coque et l'extrémité arrière de l'aile est une position classique dont on possède de nombreux exemples (cf. p. ex. Casson 1971, fig. 146, 147; Pomey 1997b, p. 84-85), mais la liberté dont il semble jouir ici, comme sur le navire des Kellia, semble être une caractéristique byzantine.

10. Pour la description technique du gréement latin et son vocabulaire, on s'appuie ici sur l'étude de René Burlet (1988).

11. Basch 1991b. Ces étais sont mentionnés dans les textes anciens sous le nom de parastatai (Casson 1971, p. 237, n. 59). Pour d'autres exemples, cf. Basch 1993, fig. 21 et fig. 23.

12. Rien ne permet ici d'identifier cet agrès à des haubans volants à bastaque
Le mât porte une grande vergue qui supporte la voile. Alors que la partie mesurable du mât correspond à la moitié de la longueur de la vergue, cette dernière, dite antenne dans le gréement latin, est égale à la longueur du navire d'étrave à étambot. L'antenne est fortement apiquée sur l'avant et est très légèrement décentrée par rapport à la tête du mât. Elle semble être monoxyle, mais on peut penser à une simplification du mosaïste. Compte tenu des dimensions supposées du navire, qui semble d'une taille relativement importante, il est possible que l'antenne soit composée de plusieurs espars, celui de la partie inférieure, vers l'avant, le quart, étant un peu plus court et un peu plus rigide que celui de la partie supérieure, vers l'arrière, la penne. Le double trait blanc à la jonction du mât et de l'antenne figure très certainement le cap de trousse qui sert à fixer l'antenne au mât. Il s'agit d'une drosse, constituée d'une estrope en boucle, qui enserre l'antenne avec le mât pour la maintenir contre ce dernier tout en lui permettant de coulisser vers le haut ou vers le bas ${ }^{13}$. De ce même point de jonction, un double cordage part vers l'arrière du navire où il se fixe à un fort palan. Il s'agit à coup sûr de la double drisse qui permet de hisser l'antenne. Les amans, qui composent cette double drisse, passent par le calcet pour redescendre vers l'arrière où ils aboutissent à un fort palan, de taille imposante, composé de deux moufles, dont le moufle inférieur est fixé vers la poupe. Ces moufles sont dénommés tailles guinderesses car le palan qu'ils constituent permet de guinder l'antenne, c'est-à-dire de la soulever. Ce dispositif caractéristique du gréement latin se retrouve sur le graffito du navire de Corinthe - où la vergue est cependant en position basse et le palan partiellement représenté - et d'une façon sensiblement identique sur la felouque des Kellia. On notera que, dans chacun de ces cas, la double drisse redescend vers la poupe où vient se fixer le palan et ne redescend pas le long du mât comme cela est habituel dans le gréement latin traditionnel. On verra là une particularité du gréement latin byzantin. En revanche l'oste, qui permet de contrôler l'extrémité supérieure de l'antenne, la penne, n'est pas représentée ici alors qu'elle est identifiée par L. Basch sur le navire des Kellia (Basch 1991b).

L'antenne porte une voile qui affecte une forme trapézoïdale en raison d'une petite chute avant beaucoup plus courte que la chute arrière (environ $1 / 8^{\mathrm{e}}$ ). Elle est fixée à l'antenne à l'aide de garcettes, disposées sur le bord d'envergure, au nombre de neuf au total, quatre sur la partie avant et cinq sur l'arrière. L'antenne et la voile sont manifestement en position longitudinale et restent dans les limites de la coque qu'elles ne débordent pas. Le navire navigue bâbord amures et compte tenu de la position de la voile, manifestement en arrière du mât, elle reçoit le vent «à bonne main», selon l'expression en usage dans le gréement latin. Sur la voile, plusieurs bandes de couleur ocre, plus foncées que le reste de la voilure traitée dans des tons rosés, descendent plus ou moins en oblique. Elles donnent du volume à la voile et suggèrent sans doute les laizes qui la composent. Mais la particularité la plus remarquable de la voile réside dans la présence de bandes de ris équipés de garcettes et destinées à permettre la réduction de la voilure. L'une de ces bandes est nettement visible dans la partie haute de la voile. Partant de la chute arrière, elle traverse la voilure en diagonale pour se terminer «à rien» sur le quart à l'extrémité avant de l'antenne. Cette bande de ris porte 17 garcettes, figurées comme autant

comme le fait L. Basch à propos de la felouque des Kellia où il est double (Basch 1991b). Sur le navire du graffito de Corinthe, il est en revanche simple, comme ici.

13. Ce dispositif de maintien de la vergue au mât est attesté dans l'iconographie où il peut figurer sous la forme d'un anneau métallique (cf. mosaïque de Khirbet Loya (Israël), Friedman 2007, fig. 1). 
de petits traits noirs verticaux. L'autre bande de ris se déduit de la réduction de la voile dans sa partie inférieure dont la bordure est enroulée sur elle-même et semble reposer sur le haut du xylokastron. La bande de ris qui a permis cette réduction devait être parallèle à la bordure inférieure de la voile, et donc être horizontale. Elle devait comporter, elle aussi, 17 garcettes, si l'on en croit le nombre de plis de l'enroulement de la voile. Autre conséquence, la réduction de la voile dans sa partie inférieure implique que lorsque la voile est totalement dépliée, elle affecte une forme trapézoïdale plus prononcée avec notamment une chute avant plus importante, à l'exemple de la voile de la petite embarcation en remorque à tribord. Enfin, on remarquera que si l'ensemble de la voile est très nettement figuré en arrière du mât, sur tribord, sa bordure inférieure repliée passe étrangement devant le mât à bâbord. Il s'agit là d'une nouvelle erreur d'interprétation du mosaïste qui s'ajoute à l'interprétation fautive de la poupe dont l'ampleur vient masquer le point d'écoute de la voile. De même sur l'avant, la proue cache le point le point d'amure de la voile dont on ne voit pas comment il est fixé.

L'étrave porte dans son prolongement un long et fin espar, sorte de «bout-dehors», qui se termine par un motif qui reprend, en plus petit, celui en forme de crosse, pointe tournée vers l'avant et portant un petit disque, qui couronne le mât principal. Or ce motif a été clairement identifié à un calcet avec sa poulie de pointe. Il s'agit donc bien d'un élément du gréement, d'un petit mâtereau, bien qu'aucun agrès ne soit figuré, autre que le calcet. Là encore, le cas n'est pas unique et le navire du graffito de Corinthe porte, de même, à l'extrémité de l'étrave, un petit mâtereau qui s'achève par un calcet à corne, dont les poulies sont bien visibles, et qui ne comporte aucun autre agrès identifiable. Notons aussi que la felouque des Kellia possède un petit gréement sur l'étrave, même si sa nature n'est pas très claire. Le type du gréement de proue du navire de la mosaïque de Kelenderis, compte tenu de sa position et de son apparente fragilité, est encore moins évident ${ }^{14}$. L'espar de l'étrave et son motif terminal ne sont donc pas liés à un élément décoratif - une hampe portant une oriflamme ou une tête d'animal - comme on aurait pu le penser au premier abord. Dès lors, le navire de la mosaïque de Kelenderis ne possède aucun élément de décoration contrairement aux bateaux du graffito de Corinthe et de la felouque des Kellia dont les mâts s'ornent d'oriflammes. Il est vrai que le jeu subtil de la polychromie du bateau lui-même suffit largement à l'effet décoratif de l'ensemble.

\section{LES EMBARCATIONS ANNEXES}

Les deux embarcations en remorque sont beaucoup plus simples et schématiques. Celle qui est remorquée à bâbord est une simple barque à deux bancs dont les extrémités, à la proue et à la poupe, sont pontées. Son extrémité avant, selon L. Casson, présenterait un tableau ${ }^{15}$. Aucun système de propulsion ni appareil de gouverne n'est figuré. Quant à l'embarcation remorquée sous voile à tribord, elle semble du même type que la précédente mais est nettement plus grande. Elle possède quatre bancs et ses extrémités sont

14. On aurait pu penser à identifier cet espar à un dastûr, sorte de bout-dehors caractéristique des voiles arabes et qui servait à avancer à l'extrême le point d'amure de la voile et à l'orienter (cf. Basch 2001, p. 65-68), mais la forme de la voile principale qui ne dépasse pas l'étrave et la position de l'espar dont l'extrémité est au-dessus du point d'amure de l'antenne l'interdisent.

15. Casson 2006. Sur les bateaux à tableau de proue, voir notamment Boetto 2009, 2014; Boetto, Poveda 2014 pontées à la proue et à la poupe. Son gréement présente de nombreuses analogies avec celui du grand bateau bien qu'il soit plus schématique. On retrouve, notamment, le même mât à calcet en forme de crosse, dont la pointe avant porte une poulie, le même étai avant et la même double drisse redescendant vers l'arrière. Mais surtout, on retrouve la bande de ris qui traverse la voile à l'horizontale dans sa partie supérieure mais qui est en biais par rapport à l'inclinaison de l'antenne. Neuf garcettes de ris sont ici visibles. Seule la géométrie de la voile est différente compte tenu du plus faible apiquage de l'antenne, à peine décentrée vers l'avant, et surtout du fait que sa partie inférieure n'est pas réduite. Du coup, la voile affecte une forme trapézoïdale plus prononcée avec notamment une chute avant importante qui reste néanmoins nettement plus petite que la chute arrière (environ 1/2).

\section{LE NAVIRE DE KELENDERIS ET LES NAVIRES DU GRAFFITO DE CORINTHE ET DU DIPINTO DES KELLIA}

$\mathrm{Au}$ cours de cette étude, nous avons vu que le navire de Kelenderis possédait plusieurs éléments en commun avec le bateau du graffito de Corinthe et celui des Kellia, comme le xylokastron et le calcet en crosse, pointe tournée vers l'avant, qui apparaissent comme autant de caractéristiques des navires byzantins. Outre la voilure principale sur laquelle nous reviendrons, il possède encore avec l'un ou l'autre de ces deux bateaux plusieurs points communs : mêmes batteries de bittes d'amarrage à l'avant du bateau de Corinthe et à l'avant et à l'arrière de celui des Kellia ; même petit mâtereau de proue à calcet en crosse que sur le bateau de Corinthe ; même système d'implantation du mât et même appareil de gouverne que sur le bateau des Kellia. Manifestement, il appartient lui aussi à la marine byzantine des Ve-VII siècle, comme l'origine de la mosaïque pouvait le laisser supposer. Son type cependant est bien différent de celui de la felouque des Kellia qui, avec son étrave et son étambot droits et ses sabords de nage, est identifié par L. Basch comme étant un léger bâtiment de guerre (Basch 1991b). En revanche, on le rapprochera volontiers du bateau de Corinthe avec lequel il possède, en plus des éléments déjà soulignés, une carène de type «symétrique », avec une proue et une poupe convexe, assez semblable, bien que le bateau de Corinthe ait une forme en croissant plus prononcée. Dans l'un et l'autre cas, il s'agit, à coup sûr de navires marchands sensiblement contemporains. On peut alors penser que le gréement du bateau de Corinthe dont la vergue, ou l'antenne, est abattue était aussi du même type.

Mais c'est à l'évidence les similitudes entre le gréement et la voilure principale du navire de Kelenderis et ceux de la felouque des Kellia qui retiennent avant tout l'attention. On retrouve la même voile en position longitudinale enverguée sur une antenne légèrement décentrée sur l'avant et fortement apiquée et le même système de double drisse passant dans un calcet en crosse pour redescendre sur l'arrière et aboutir à un fort palan à deux moufles, les tailles guinderesses. Tous ces éléments sont caractéristiques du gréement latin et L. Basch n'hésite pas à qualifier la voile de la felouque des Kellia de voile latine «sans la moindre équivoque» (Basch 2001, p. 57). Cependant, la voile de Kelenderis n'est pas parfaitement triangulaire comme la voile latine classique. Elle possède encore une chute avant importante qui lui confère une forme trapézoïdale prononcée. Or, il est très vraisemblable qu'il en soit de même pour la voile des Kellia dont la partie avant n'est pas complètement dessinée. Si l'on considère, en effet, l'orientation de la bordure 


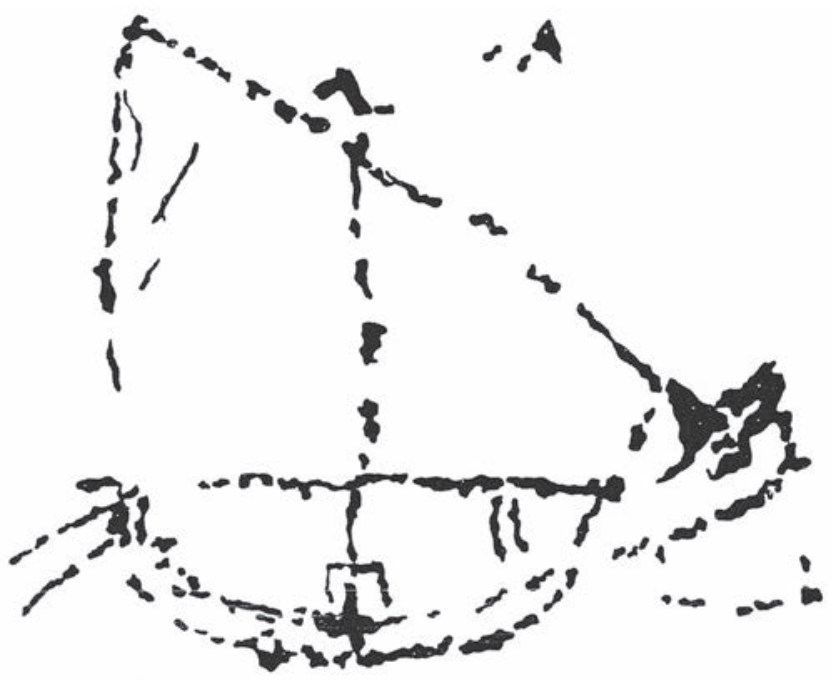

Fig. 8 : Dipinto d'un bateau à voile latine trapézoïdale provenant d'une maison d'Alexandrie (fin du vies.) (Basch 1993).

inférieure de la voile, celle-ci ne rejoint pas l'extrémité avant de l'antenne et l'on peut admettre la présence d'une chute avant. On serait alors en présence, ici aussi, d'une voile trapézoïdale et non pas d'une voile latine classique comme le pense L. Basch. Un autre exemple de voile trapézoïdale avec une petite chute avant est donné par un dipinto de bateau tracé sur le mur d'une maison d'Alexandrie de la fin du VI $^{\mathrm{e}}$ siècle $^{16}$ (fig. 8).

En revanche, la différence essentielle entre les voilures des navires de Kelenderis et des Kellia tient dans la présence, sur la voile de Kelenderis, de bandes de ris dans la partie supérieure et inférieure de la voile. Ces bandes de ris, notamment la bande supérieure disposée en biais, renvoient indiscutablement, là encore, à un gréement latin. Elles n'ont plus rien à voir avec le système de cargues du gréement carré antique dont le souvenir est peut-être rappelé par le quadrillage de la voile de la felouque des Kellia ${ }^{17}$. La représentation de telles bandes de ris est tout à fait exceptionnelle et, à ma connaissance, il s'agit là de la plus ancienne attestation de ces manœuvres ${ }^{18}$. Leur présence implique un gréement latin déjà évolué, fruit d'une longue tradition et d'une longue pratique, quand on sait les difficultés et le danger, souligné par R. Burlet, d'une prise de ris antenne en l'air (Burlet 1988). C'est pourquoi, la manœuvre avait lieu généralement après avoir amené l'antenne sur le pont. C'est peut-être aussi pour pallier ces inconvénients que l'on a adopté sur le navire de Kelenderis une bande de ris disposée horizontalement dans la partie inférieure de la voile comme le permet la forme trapézoïdale de la voile. Cette disposition sera en fait une caractéristique de cette forme particulière de voile latine, précisément pour faciliter la prise du premier ris, et est encore en usage de nos jours ${ }^{19}$.

16. Basch 1993, fig. 23. Le rapprochement est d'autant plus intéressant que le bateau possède la même implantation du mât à l'aide d'étais verticaux.

17. Pour autant, il est peu probable que l'on ait encore un système de cargues sur la voile du bateau des Kellia, ce système étant peu compatible avec une voile axiale asymétrique.

18. Jusqu'à présent de telles bandes de ris ne semblaient pas être en usage en Méditerranée avant le $\mathrm{XVI}^{\mathrm{e}} \mathrm{s}$. La plus ancienne représentation jusqu'alors connue provenait du domaine atlantique et était fournie par le navire du sceau de La Rochelle (v. 1200) qui porte une voile carrée avec trois bandes de ris. Je dois ces renseignements à mon ami Eric Rieth que je tiens à remercier.

19. La présence de bandes de ris horizontales situées dans la partie inférieure de la voile, souvent associées à des bandes de ris courant en biais dans la partie supé-
Ainsi, avec le navire de la mosaique de Kelenderis, on se trouve en présence, dans la première moitié du ve siècle ap. J.-C., d'un navire byzantin muni indiscutablement d'un gréement latin déjà très évolué et possédant pratiquement tous les éléments caractéristiques de la voile latine, notamment dans le gréement dormant et le gréement courant. Seule la géométrie de la voile de forme trapézoïdale - même si elle peut affecter une forme pratiquement triangulaire lorsque la partie inférieure de la voile est réduite - interdirait de parler de «voile latine» stricto sensu. Pour autant, on se gardera bien de parler de «voile arabe» comme sa forme trapézoïdale pourrait y inviter. Compte tenu de la datation de la mosaïque, cela n'aurait aucun sens et constituerait un anachronisme. En outre, L. Basch a montré très justement qu'il n'y avait guère de relation entre la voile latine méditerranéenne et la voile arabe de l'Océan Indien $^{20}$. La voile trapézoïdale du navire de Kelenderis est souvent appelée «voile de mistic» par référence à un type de navire moderne méditerranéen portant ce gréement (Beaudouin 1990, p. 31). Cependant, toujours par souci d'éviter tout anachronisme, on préférera parler de «voile latine orientale», par référence à son origine de Méditerranée orientale, comme le propose F. Beaudouin (Beaudouin 1990, p. 27) ${ }^{21}$. Par rapport à la voile latine triangulaire classique, la voile latine trapézoïdale est d'un maniement plus simple qui cependant n'autorise pas autant de réglages. Elle ne possède ni davant ni orse poupe et de ce fait s'amure à poste fixe. Il est dommage, le point d'amure étant masqué, que l'on ne puisse pas vérifier ce détail important et caractéristique sur le navire de Kelenderis.

Ainsi, par sa datation dans la première moitié du v $v^{e}$ siècle et par ses caractéristiques, le navire de la mosaïque de Kelenderis apporte des données nouvelles et fondamentales au dossier de la famille des voiles latines et de leur origine méditerranéenne ${ }^{22}$.

\section{LE «PROCESSUS D’AURICISATION »}

Antérieur d'environ deux siècles à la felouque des Kellia, qui restait à ce jour le plus ancien témoignage assuré d'une voile de type latin en Méditerranée, il confirme de façon indiscutable l'origine purement méditerranéenne de la voile latine et son indépendance par rapport à la voile arabe originaire de l'Océan Indien démontrée par L. Basch.

rieure, est une caractéristique de ce gréement latin trapézoïdal, que l'on trouve notamment sur les mistics (cf. p. ex. le mistic la Vierge des Carmes, par Nicolas Camillieri (déb. XIX ${ }^{\mathrm{e}}$ s.) (Cadoret et al. 2009, p. 204). On retrouve encore aujourd'hui, sur le Ville-de-Fréjus, pointu construit par R. Autiéro, la même disposition des bandes de ris associées à une voile latine trapézoïdale à chute avant: en haut, une bande de ris en biais qui se termine «à rien» du quart; en bas, une bande de ris horizontale, parallèle à la bordure inférieure de la voile (Vigne 1994; Huet et al. 2004, p. 31).

20. Basch 2001. Bien que la Méditerranée ait connu la voile latine triangulaire et la voile latine trapézoïdale que l'on ne confondra pas pour autant avec la voile arabe, contrairement à ce que laisse entendre E. Rieth (2016), ce dernier reprend à juste titre l'idée que la Méditerranée et l'Océan Indien ont constitué deux espaces maritimes indépendants sur le plan nautique. J'étais arrivé à la même conclusion concernant les techniques de construction navale et notamment les systèmes d'assemblage par ligatures (Pomey 2011).

21. Sur la question de la terminologie cf. Whitewright 2009.

22. L. Basch $(1997,2001)$ a réuni un important et très complet dossier sur cette question avant la découverte de la mosaïque de Kelenderis. On lira aussi avec intérêt son étude sur les bateaux coptes (Basch 1993). Voir plus récemment Polzer 2008; Whitewright 2009, 2011. 


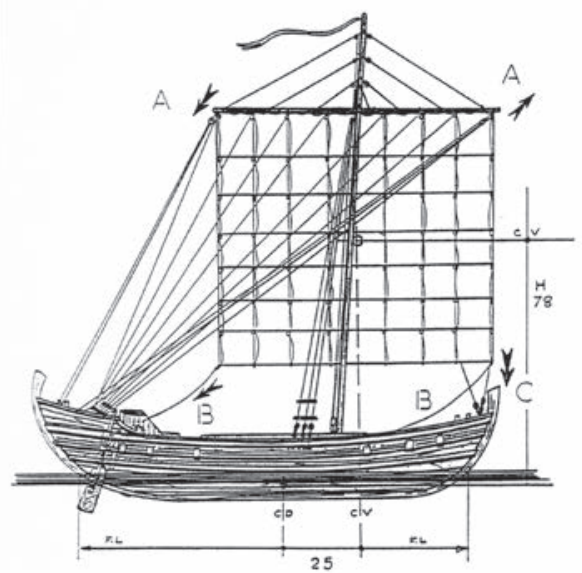

a. Voile de portant avec vergue brassée en pointe. Toile maximum.

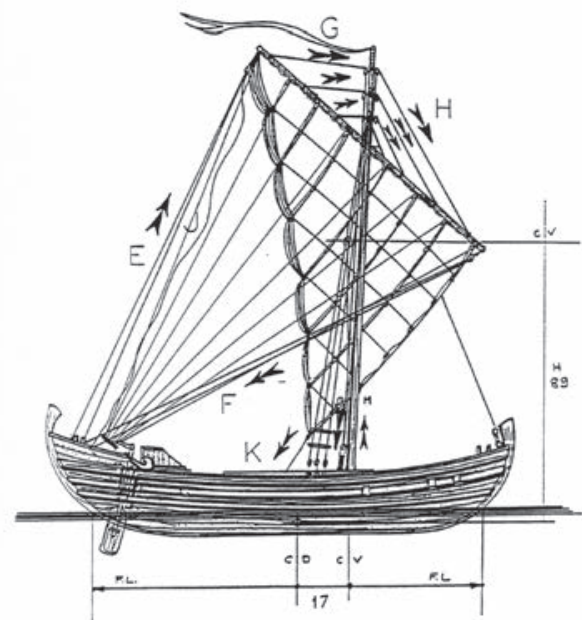

c. Voilure de près par une force de vent moyenne 4 à 5 beaufort. Toile réduite de moitié, vergue fortement inclinée sur lavant.

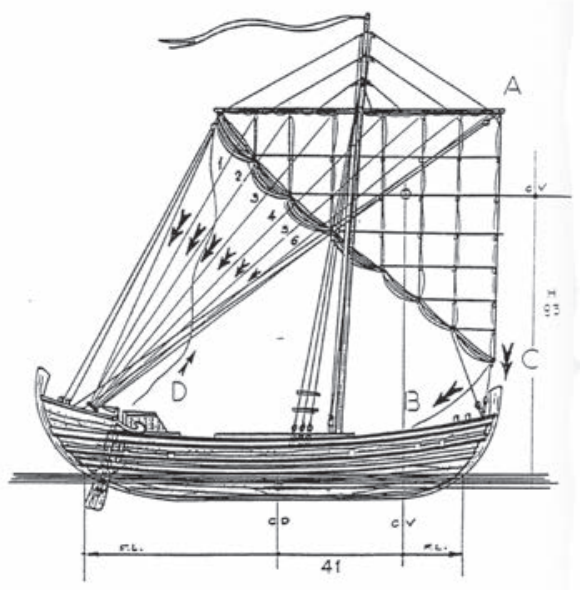

b. Voilure de transition portant-près. Toile réduite de moitié.

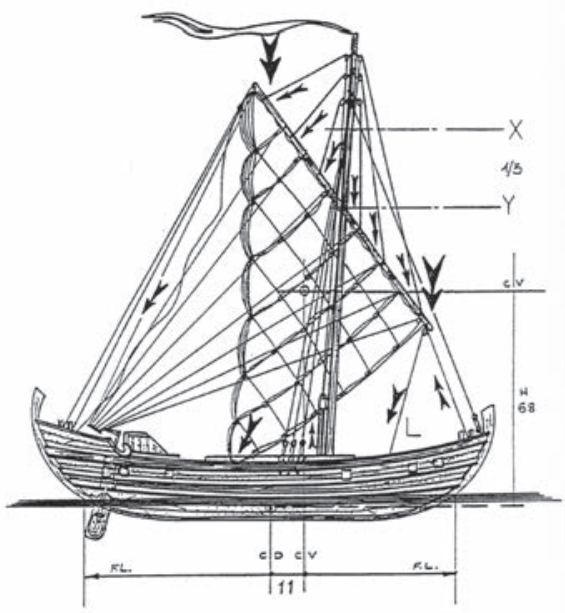

d. Voilure de près par vent relativement fort, toile réduite de moitié, vergue fortement inclinée sur l'avant, puis amurée plus bas sur le mât d'un tiers (centre de voilure abaissé et reculé).

Fig. 9: Schéma du processus d'auricisation permettant de passer d'une voile carrée à une voile triangulaire axiale, type voile latine. (Dessin R. Roman, Aix Marseille Univ, CNRS, Minist Culture \& Com, CCJ, Aix-en-Provence, France).

Par la forme de sa voilure, trapézoïdale, il confirme que la voile latine trouve bien son origine dans l'auricisation de la voile carrée antique, comme l'ont affirmé plusieurs auteurs ${ }^{23}$. Mais par son degré d'évolution très avancé, notamment par la présence de bandes de ris, il montre que ce processus d'évolution est déjà engagé depuis longtemps et que la pratique du gréement latin, c'est-à-dire comportant une voile longitudinale asymétrique enverguée sur une antenne apiquée avec les agrès correspondant, bénéficie déjà d'une longue expérience acquise au cours d'une période de «stabilisation technique» (Whitewright 2011, p. 98-99).

Comme l'a démontré F. Beaudouin (1990, p. 27-31), et pour reprendre son expression, le «processus d'auricisation» de la voile carrée a pour origine la manœuvre longitudinale de la voile qui conduit à la modification de la position du mât (déplacement ou inclinaison), au décentrage du point de drisse et à la déformation de la géométrie de la voile qui devient asymétrique. Le problème réside alors dans la nécessité d'ajuster le centre de voilure au centre de dérive latérale. Compte tenu de l'importance du plan de dérive

23. Voir par exemple: Casson 1966, 1971, p. 276-277; Adam 1970; Beaudouin 1990, p. 27-31 ; Basch 1997, 2001; Pomey 1997a, 1997b ; Polzer 2008. de l'appareil de gouverne des navires antiques, l'une des solutions est alors le basculement de la voile à la géométrie modifiée par l'apiquage de la vergue qui a pour effet de reculer le centre de voilure. Ce sont ces modifications, qui peuvent se manifester indépendamment les unes des autres comme le rappelle F. Beaudouin, qui vont donner naissance, une fois adoptées et fixées, à la voile latine. Ce processus d'auricisation s'accompagne, en outre, d'une profonde modification des différents agrès courants et dormants qui servent à manœuvrer la voile et que la pratique va finir par imposer $^{24}$.

L. Basch a montré, à travers des exemples de graffiti de Délos, que l'apiquage de la vergue était déjà pratiqué au moins dès le II $^{\mathrm{e}}$ siècle av. J.-C. Le célèbre texte d'Aristote (ou plutôt du PseudoAristote), Mechanica, 851 b, qui décrit la manœuvre que l'on doit faire lorsque l'on veut poursuivre sa route par vent défavorable et qui consiste à brasser la vergue pour l'amener en position axiale, puis à carguer la voile dans sa partie arrière, constitue à l'évidence un bel exemple de processus d'auricisation tel que décrit par F. Beaudouin.

24. Sur l'importance de l'évolution des agrès qui accompagne l'évolution de la géométrie de la voile en raison de nouvelles pratiques, cf. Whitewright 2009, 2011. 
En effet, la voile supposée carrée est brassée pour venir en position longitudinale et sa géométrie est modifiée pour devenir triangulaire. Or, explique Aristote, cette manœuvre a pour but de rendre au gouvernail toute son efficacité en utilisant la mer comme point de levier. C'est là un détail important, sur lequel insiste F. Beaudouin mais rarement souligné dans l'analyse de ce texte. Il vient confirmer que l'on est en présence d'une manœuvre d'auricisation qui a pour objectif d'ajuster le centre de voilure au centre de dérive latéral. Or, sur les navires antiques, ce dernier centre était directement dépendant du gouvernail latéral du fait de leur surface importante. C'est bien cette particularité que souligne Aristote en insistant sur la relation existant entre la modification de la voilure et l'efficacité du gouvernail. Contrairement à la lecture de L. Basch, qui voit dans l'opération une simple manœuvre de cap courante ${ }^{25}$, on est ici en présence d'une question théorique - comment naviguer contre le vent? - qui se traduit par un problème mécanique dont la solution est dans la transformation de la géométrie de la voilure mise en position longitudinale. Pour que la manœuvre soit efficace, il faudrait aussi que la vergue soit apiquée pour reculer le centre vélique. Il est vrai que cette opération n'est pas décrite explicitement par Aristote - sans doute était-elle implicite et induite par la manœuvre ellemême - mais elle est dans la logique de la solution mécanique proposée. Un passage du roman d'Achille Tatius, Leucippé et Clitophon (3.1.1), datant vraisemblablement du $\mathrm{II}^{\mathrm{e}}$ siècle ap. J.-C., décrit à son tour la même manœuvre (Casson 1971, p. 276-277 ; Rougé 1978, p. 273-276). Pour L. Casson, la transformation de la voile carrée en voile triangulaire axiale au cours des manœuvres décrites par Aristote et Achille Tatius, serait à l'origine de la voile latine selon une interprétation que j'ai moi-même suivi en l'illustrant le plus précisément possible ${ }^{26}$ (fig. 9).

Qu'il s'agisse de la manœuvre décrite par Aristote et Achille Tatius ou des navires des graffiti de Délos, on ne peut pas encore parler de voile latine: la voile est carrée et les agrès sont ceux du gréement carré (bras, cargues et écoutes essentiellement). Mais dans tous ces cas, nous sommes bien en présence d'un processus d'auricisation. Et c'est très vraisemblablement dans ce processus, entamé depuis longtemps dans certaines circonstances, qu'il convient de chercher l'origine de la voile latine méditerranéenne.

Il reste un dernier point à éclaircir, celui de l'apparition de la voile latine purement triangulaire. Nous avons vu que sur la mosaïque de Kelenderis la voile est encore trapézoïdale et qu'il en était sans doute de même sur la felouque des Kellia. À ces deux documents, on peut aussi ajouter le dipinto d'Alexandrie de la fin $\mathrm{du} \mathrm{VI}^{\mathrm{e}}$ siècle que nous avons déjà évoqué, et vraisemblablement la stèle funéraire d'Alexandre de Milet (II ${ }^{\mathrm{e}}$ s. ap. J.-C., aujourd'hui au Musée archéologique du Pirée). Le gréement du petit bateau qui figure sur cette stèle a souvent été interprété comme un exemple de voile latine orientale dont il serait le plus ancien témoignage

25. Basch 1997 , p. $216-217 ; 2001$, p. 58-60. Une telle manœuvre de mise à la cap courante aurait peu d'intérêt du point de vue théorique et purement mécanique dans lequel se place Aristote.

26. Casson 1971, p. 276, n. 24, fig. 188; Pomey 1997a, p. 97-99; 1997b, p. 80-82. On pourra objecter que les balancines figurées sur les dessins (cf. supra fig. 9) n'étaient pas en usage à l'époque d'Aristote puisqu'elles n'apparaîtront sur les navires gréco-romains qu'au cours du II $^{\mathrm{e}} \mathrm{s}$. av. J.-C. Si elles figurent, c'est parce que le navire pris pour modèle est le navire des Laurons II, dont l'état de conservation exceptionnel de la coque et la présence du massif d'emplanture (Gassend et al. 1984) permettait de calculer avec précision le centre de dérive et le centre de voilure. Or à l'époque de ce navire (III ${ }^{\mathrm{e}} \mathrm{s}$. ap. J.-C.), les gréements carrés comportaient des balancines. Rappelons que sur les petites unités, les balancines ne sont pas indispensables à l'apiquage de la vergue et que, sur certains graffiti de Délos, les vergues apiquées en sont dépourvues (Basch 1989, 2001). (fig. 10) ${ }^{27}$. J. Whitewright, tout en admettant la présence d'une voile latine trapézoïdale (settee sail), est le seul à avoir noté l'absence de toute représentation d'agrès caractéristiques de ce gréement et en déduit qu'il s'agirait d'une représentation précoce du gréement latin antérieure à la standardisation du gréement et de ses manœuvres (Whitewright 2009, p. 102). Cependant cette interprétation du gréement du bateau de la stèle d'Alexandre de Milet repose sur la présence d'une vergue curviligne fortement inclinée sur l'avant et sur les deux lignes verticales qui descendent des extrémités de la vergue et qui figureraient les bords de chute de la voile. En revanche, on notera que la bordure de fond de la voile n'est pas représentée. Dès lors, les lignes verticales descendant des extrémités de la vergue pourraient être, tout aussi bien, les bras de manœuvre de la vergue d'un gréement carré. L'inclinaison du mât, et la position fortement apiquée de la vergue et sa forme curviligne seraient alors dû à la contrainte du champ décoratif qui affecte dans sa partie supérieure une forme en arc de plein cintre ${ }^{28}$. Dès lors, si l'interprétation du gréement du bateau de la stèle d'Alexandre de Milet comme étant une voile latine orientale reste très possible, il ne peut pour autant constituer un document indiscutable.

On peut penser que la réduction de la voilure latine orientale trapézoïdale qui devait s'effectuer par le bas, comme on le voit sur la mosaïque de Kelenderis, est un processus qui aurait pu conduire à l'adoption de la voile latine triangulaire. Mais L. Basch fournit l'exemple d'un dipinto, figuré dans un hypogée de la nécropole d'Anfouchi à Alexandrie, représentant une petite embarcation munie d'une pure voile latine triangulaire qu'il date probablement du $\mathrm{I}^{\text {er }}$ siècle av. J.-C., voire «pas au-delà du règne d'Auguste» (fig. 11) ${ }^{29}$. Malheureusement, si l'interprétation est indiscutable, la datation reste incertaine. Quoi qu'il en soit, il s'agit manifestement d'une petite embarcation légère et il y a tout lieu de penser, que la voile latine triangulaire soit d'abord apparue sur de petites embarcations avant d'être adoptée sur des unités plus importantes. Le problème serait donc plus complexe et moins linéaire que l'on aurait pu le penser et l'on aurait en somme une double évolution: l'une concernant de petites embarcations et la voile latine triangulaire; l'autre intéressant des navires plus importants et la voile latine orientale trapézoïdale. Evolutions parallèles et non exclusives puisqu'aux $\mathrm{V}^{\mathrm{e}}$ et $\mathrm{VI}^{\mathrm{e}}$ siècles et sans doute encore au $\mathrm{VII}^{\mathrm{e}}$, la voile latine orientale reste encore largement en usage notamment sur les bâtiments byzantins d'une certaine importance comme le navire de Kelenderis ou la felouque des Kellia. Cependant, ce développement de la voile latine orientale n'est pas sans soulever de nombreuses interrogations. Alors qu'elle semble avoir atteint un degré d'évolution important dont témoigne la présence des bandes de ris, à la fois horizontale dans le bas de la voile et oblique dans sa partie

27. Casson 1966, fig. 4; 1971, p. 244 et fig. 181; Baudoin 1990, p. 31. Je tiens à remercier ici Katerina Dellaporta pour m'avoir fourni des photographies inédites de ce relief.

28. L. Basch (1997, p. $219 ; 2001$, p. 61-62) interprète de cette façon la vergue du bateau de la stèle d'Alexandre de Milet, mais considérant les lignes verticales comme les bords de chute de la voile, il voit dans cette dernière une voile au tiers qui n'existant pas dans l'Antiquité serait une erreur de l'artiste due à des raisons esthétiques.

29. Basch 1987, fig. 1084; 1989, fig. 8 ; 1993, fig. 22 ; 1997, fig. 11 ; 2001, fig. 9. La datation repose sur l'analogie de style avec des dipinti voisins représentant des navires de type hellénistique. Pour une datation au plus tard sous Auguste, cf. Basch 1991 b, p. 10. On ne retiendra pas, en revanche, le graffito représentant un bateau à voile latine triangulaire qui figure sur un fragment de tuile provenant de Thasos (Casson 1966, p. 58, fig. 5; 1971, fig. 180; Polzer 2008, p. 245, fig. 20). L'origine précise et la date de ce graffito restent en effet inconnues et une datation tardive à l'époque byzantine apparaît très probable (Rougé 1978, p. 275 ; Basch 1997, p. 219 ; 2001, p. 62). 


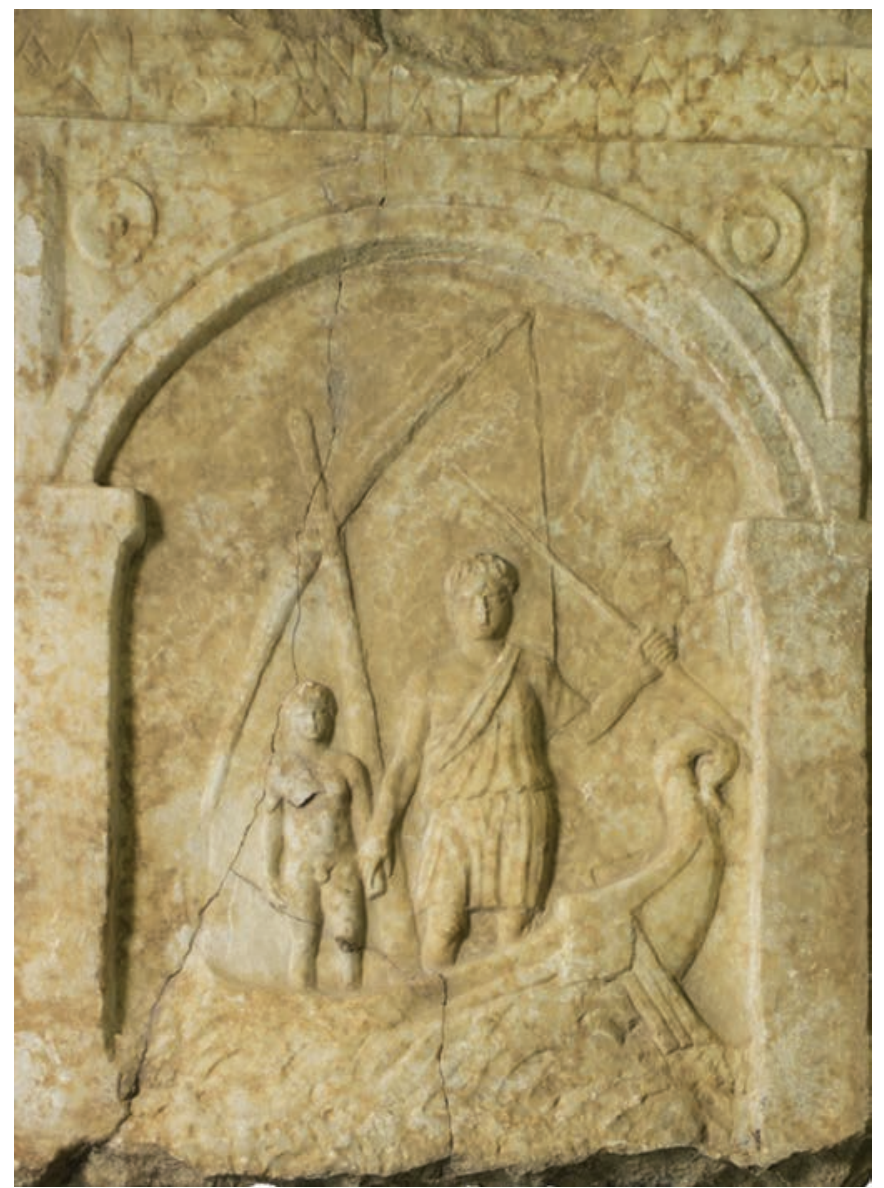

Fig. 10: Stèle d'Alexandre de Milet (II S. ap. J.-C., Musée archéologique du Pirée) (Photo K. Dellaporta).

supérieure, cette voile disparaît ensuite de la documentation, et notamment de l'abondante iconographie byzantine, jusqu'à l'époque moderne. Il semble même qu'il faille réinventer certains aspects techniques comme la bande de ris supérieure oblique qui ne réapparaîtra que plus tard dans la seconde moitié du XVII'. S'agit-il d'un problème de sources et d'un effet des lacunes de notre documentation, ou d'un problème de «régression technique», suivi d'une réintroduction de ce type de gréement - par une éventuelle influence de la voile arabe, originaire de l'Océan Indien - et une réinvention technique? La question reste posée et montre que les problèmes soulevés par la famille des voiles latines, et notamment de la voile latine orientale, sont loin d'être totalement résolus ${ }^{30}$.

\section{LES STEREOMETRICA (II, 48-49)}

Arrivé à ce stade de la discussion, il convient de verser au dossier un nouveau document, non pas inédit, mais jusqu'à présent totalement ignoré du point de vue nautique. Il s'agit d'un passage des Stereometrica d'Héron d'Alexandrie concernant une formule simple permettant de calculer le nombre de pièces de toile de dimensions connues entrant dans la confection d'une voile dont on connaît deux des côtés. Ce qui revient à en calculer la surface.

30. Là encore, je tiens à remercier Eric Rieth pour avoir attiré mon attention sur ces questions.
Stereometrica (II, 48-49):

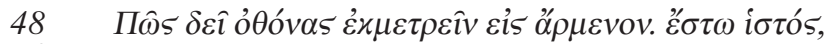

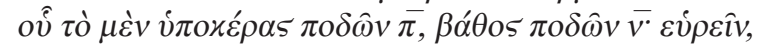

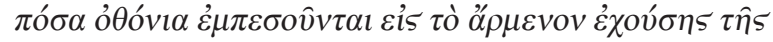

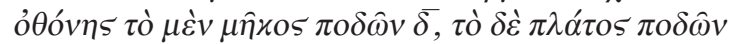

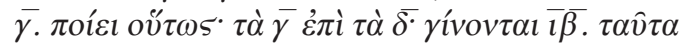

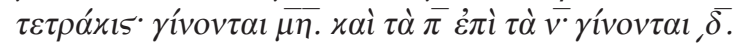

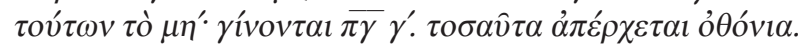

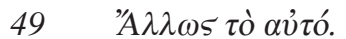

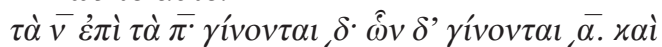

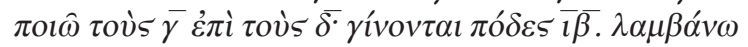
$\tau \hat{\omega} v, \bar{\alpha} \tau o ̀ ~ \imath \beta^{\prime} \cdot \gamma^{\prime} v o v \tau \alpha \imath \overline{\pi \gamma} \gamma^{\prime} . \varphi \alpha v \varepsilon \rho o ́ v$.

(texte établi par Heiberg 1976, p. 128).

\section{Traduction :}

48. Comment mesurer la toile destinée à une voile. Soit un gréement dont l'antenne est de 80 pieds, la profondeur de 50 pieds; pour trouver combien de pièces de toile peuvent entrer dans la voile, sachant qu'une pièce a 4 pieds de hauteur et 3 pieds de largeur, faire ainsi: 3 par 4 donne 12; ce résultat multiplié par 4 donne 48 ; 80 par 50 donne 4000; ce résultat divisé par 48 donne 83 1/3. C'est le nombre de pièces de toile qui convient.

49. De même, d'une autre façon:

50 par 80 donne 4000; divisé par 4 cela donne 1000 ; je fais ensuite 3 par 4, cela donne 12 pieds [carrés] ;

je prends 1000 que je divise par 12, cela donne $831 \frac{1}{3}$. Ce qui est évident.

Soit, en résumé: $3 \times 4=12 ; 4 \times 12=48 ; 80 \times 50=4000$; $4000 / 48=83 \frac{1}{3}$;

ou encore: $50 \times 80=4000 ; 4000 / 4=1000 ; 3 \times 4=12$ pieds; $1000 / 12=83 \frac{1}{3}$.

On notera que si le terme $\not ́ \rho \mu \varepsilon v o v$ désigne ici la voile, i $\sigma \tau o ́ \varsigma$ se

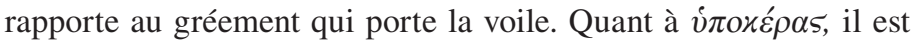
l'équivalent de $\chi \varepsilon ́ \rho \alpha \varsigma$ et désigne l'antenne supportant la voile, ou plus précisément le bord d'envergure de cette dernière, c'est-à-dire

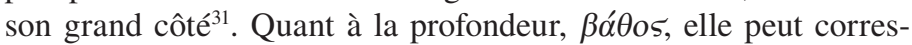
pondre aussi bien à la hauteur qu'à la largeur de la voile. En outre, le texte de l'édition Teubner est accompagné d'un dessin représentant un mât avec une voile latine triangulaire en forme de triangle rectangle (fig. 12).

\section{CALCUL DE LA SURFACE DE LA VOILE}

Selon la formule des Stereometrica: on calcule tout d'abord la surface d'une pièce de toile entrant dans la confection de la voile,

31. Je tiens à remercier Mme Micheline Decorps-Foulquier, professeur émérite à l'Université de Clermont-Ferrand, MM. Philippe Fleury, professeur à l'Université de Caen, et Jean-Yves Guillaumin, professeur à l'Université de Franche-Comté, spécialistes des textes scientifiques grecs et d'Héron d'Alexandrie, pour leurs conseils avisés et leurs remarques précieuses sur l'établissement du texte et sa traduction ainsi que M. Antoine Hermary, professeur émérite à l'université d'Aix Marseille, pour avoir bien voulu relire le texte grec. Pour le vocabulaire, voir aussi Casson 1971, p. 232 , n. 35 et p. 233 . 

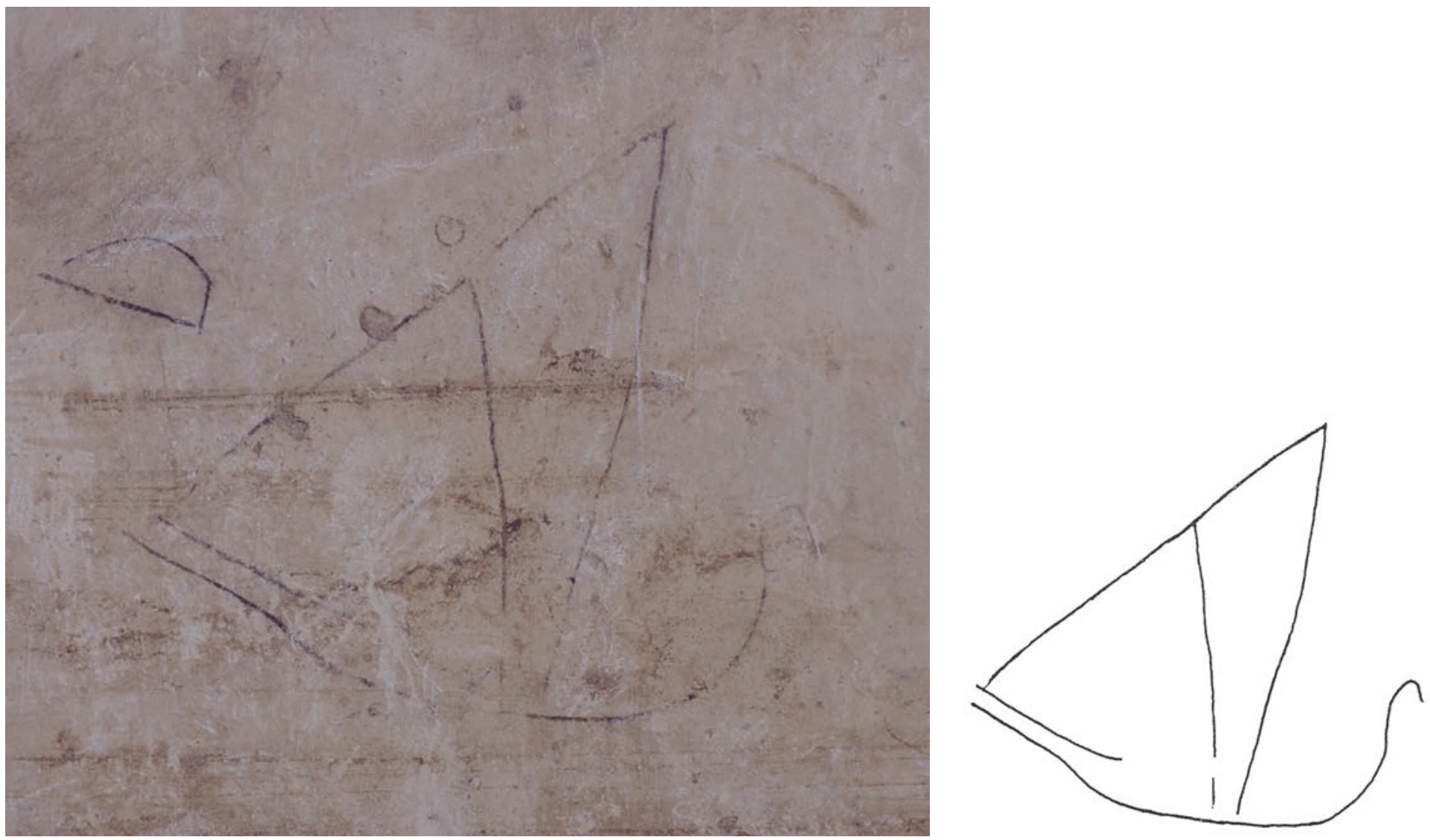

Fig. 11: a - Dipinto d'Anfouchi représentant une petite embarcation à voile latine (v. er siècle av. J.-C., Alexandrie) (Photo A. Pelle, CEAlex, CNRS). $b$ - Relevé du dipinto d'Anfouchi (v. ${ }^{\text {er }}$ siècle av. J.-C., Alexandrie) (Dessin L. Basch).

soit $3 \times 4=12$ pieds $^{2}$; puis on calcule la surface du rectangle construit sur les deux dimensions de la voile, soit 50 × $80=$ 4000 pieds $^{2}$; enfin, cette surface est divisée par 4 fois la surface d'une pièce de toile, soit $4000 /(4$ x 12$)=83 \frac{1}{3}(83,333)$.

La question est de savoir pourquoi la formule d'Héron passe par le rectangle construit sur les deux dimensions connues de la voile, puis par sa division par un facteur $4^{32}$.

À l'évidence, il ne peut s'agir d'une voile rectangulaire puisqu'il suffirait alors de diviser la surface du rectangle par celle des pièces de toile. La division supplémentaire par 4 n'aurait de fait aucun sens. Il ne peut guère plus s'agir d'une voile en forme de triangle rectangle, comme le suggère le dessin accompagnant le texte de l'édition Teubner, puisque, dans ce cas, il suffirait de diviser la surface par 2 et non par 4. On en déduira que le dessin d'illustration du texte a été vraisemblablement corrompu par les copistes $^{33}$. Cette incompatibilité entre le texte et son illustration a été relevée par Heiberg (1976, p. 128, n. **) qui suggère une autre forme de voile avec un bord de fuite curviligne dont le calcul de la surface serait alors empirique. On notera que ce dernier type de voile dont il donne le schéma (fig. 13) n'est pas attesté dans l'Antiquité, ni à l'époque byzantine, ni au Moyen Âge. Cette solution ne peut donc être retenue.

La seule possibilité est qu'il s'agit d'une voile triangulaire

32. Je tiens aussi à remercier Pierre Poveda, Ingénieur de recherche CNRS au Centre Camille Jullian, pour m'avoir aidé à élaborer la solution graphique de ce problème.

33. Selon Heiberg 1976, Prolegomena, IV, toutes les figures viennent du manuscrit du Vieux Sérail (Seragliensis gr. 1), codex (S) de Constantinople, Constantinopolit. palatii veteris $\mathrm{n}^{\circ} 1$, du $\mathrm{X}^{\mathrm{e}}-\mathrm{XI}^{\mathrm{e}} \mathrm{s}$. Voir aussi Vitrac 2010 quelconque (triangle scalène) avec un grand côté d'une longueur de 80 pieds correspondant au bord d'envergure, côté antenne, et un côté de 50 pieds correspondant à la «profondeur». Notons que cette profondeur peut correspondre aussi bien au bord de chute de la voile et dans ce cas indiquer sa hauteur, comme elle peut correspondre au bord de fond et indiquer sa largeur. Dès lors, connaissant la surface du triangle par la formule des Stereometrica qui est égale à 1000 pieds $^{2}$, il est facile de calculer la longueur du troisième côté qui est alors de 44,40 pieds.

La formule consiste donc à diviser par 4 le rectangle construit sur les deux longueurs principales de la voile. Ce qui est facile à obtenir en divisant le rectangle par ses deux diagonales. On obtient ainsi 4 triangles isocèles de surface égale correspondant bien au 1/4 de la surface totale (surface rectangle $50 \times 80=4000$ pieds $^{2} ; 1 / 4$ surface $=$ surface des triangles $=1000$ pieds $^{2}$ ). Deux de ces triangles isocèles ont bien une base de 80 pieds mais leurs deux côtés égaux mesurent alors 47,17 pieds (fig. 14). On voit que pour une même surface et une même base, la différence entre les deux autres cotés du triangle d'origine et des triangles isocèles obtenus par la division par 4 est très faible: 47,17 pieds au lieu de 50 p. et 44,40 p. Soit une différence par défaut de 2,83 pieds dans un cas et par excès de 2,77 pieds dans l'autre. Ce qui correspond à $0,94 \%$ des longueurs des côtés du triangle d'origine, ce qui est tout à fait acceptable ${ }^{34}$.

On le voit, la formule est d'une grande simplicité - et donc facile

34. On aurait aussi pu penser à une voile en forme de triangle isocèle du type sipharos/suparum, située en tête de mât au-dessus de la vergue de la voile carrée principale, et caractéristique des grands navires alexandrins (Casson 1971, p. 241242). Mais dans ce cas, la formule ne s'applique pas. La surface du triangle isocèle 


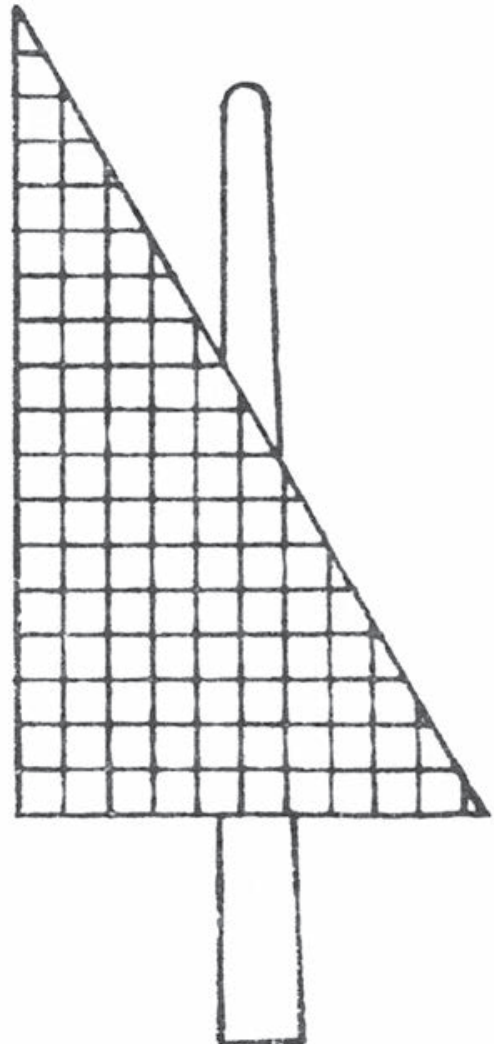

Fig. 12: Schéma de la voile latine illustrant les Stereometrica (II, 48-49) d'Héron d'Alexandrie (Heiberg 1976, p. 128).

à appliquer - et donne un résultat exact et précis en surface en passant par des triangles isocèles de dimensions très proches du triangle d'origine.

L'existence d'une telle formule dans les Stereometrica d'Héron d'Alexandrie pourrait laisser supposer que la voile latine triangulaire était d'usage fréquent à Alexandrie dans la seconde moitié du $\mathrm{I}^{\text {er }}$ siècle de notre ère, si tant est que l'on admette que le grand mathématicien a bien vécu à cette époque et que l'on puisse lui attribuer les Stereometrica. Ce qui est loin d'être évident, Héron d'Alexandrie étant malheureusement, selon B. Vitrac, «le plus problématique des auteurs d'écrits mathématiques grecs»(Vitrac 2010 , p. 1)! Selon ce dernier, le corpus héronien rassemblé par Heiberg sous le titre de Stereometrica a été entièrement reconstitué à partir d'extraits figurant dans de nombreux manuscrits dont aucun ne contient la collection finale (Vitrac 2010, p. 16-21). Les textes la composant seraient d'origines diverses, les uns tardifs et d'époque byzantine, d'autres pouvant dater des $I^{\mathrm{e}}-\mathrm{V}^{\mathrm{e}}$ siècles, voire même avant et être contemporains d'Héron.

\section{ALEXANDRIE, CENTRE D'INNOVATION ET DE DIFFUSION DES TECHNIQUES NAUTIQUES}

Néanmoins, on retiendra que ces textes témoignent de la profonde influence de l'œuvre héronienne qui s'inscrit elle-même directement dans le contexte alexandrin. Si la date et l'origine du

ayant une base de 80 p. et des côtés de 50 p. serait de 1200 pieds $^{2}$ au lieu de 1000 et il faudrait 100 pièces de toile au lieu de $83 \frac{1}{3}$.

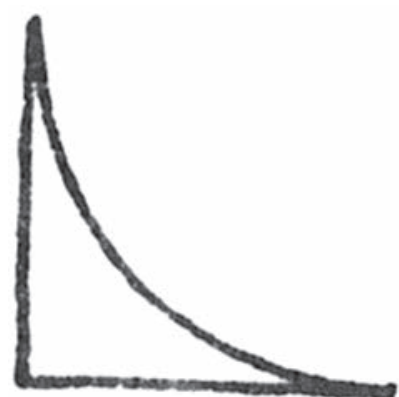

Fig. 13: Schéma de voile triangulaire à bord curviligne proposé par Heiberg (Heiberg 1976).

texte qui nous intéresse restent malheureusement indéterminées, son sujet même qui accorde à la voile latine triangulaire un intérêt particulier peut cependant constituer un indice supplémentaire du rôle qu'aurait joué la ville d'Alexandrie dans le développement des techniques nautiques et de la voile latine en particulier. Siège de la plus importante école scientifique qu'a connue le monde antique, située à l'interface entre le Nil, le lac Mariout et la Méditerranée, dépositaire des traditions ancestrales de la navigation fluviale et maritime, Alexandrie et son complexe portuaire fluvio-maritime aux multiples bassins possède tous les éléments pour être un grand centre d'innovation et de diffusion des techniques nautiques. Le texte des Stereometrica viendrait ainsi en soutien de l'hypothèse de L. Basch qui voyait dans le grand port l'endroit idéal pour de nouvelles expériences navales, dont la voile latine (Basch 1997, p. 219220 ; 2001, p. 63). Au dessin d'Anfouchi s'ajoute en effet un autre dipinto, daté probablement $\mathrm{du} \mathrm{VI}^{\mathrm{e}}$ siècle, provenant d'une habitation alexandrine et représentant un bateau à voile latine orientale (cf. supra, fig. 9 et 11). On pourrait aussi citer la célèbre lettre de Synésius (Epist. 4) relatant au tout début du ve siècle son voyage maritime d'Alexandrie en Cyrénaïque. Selon L. Casson le bateau alexandrin sur lequel il a embarqué serait muni d'une voile latine (Casson 1966, p. 49; 1971, p. 268-269). De fait, les jalons ne manquent pas en Égypte et, à ces exemples alexandrins, on peut ajouter un autre exemple de bateau à voile latine du VII ${ }^{\mathrm{e}}$ siècle provenant des Kellia (fig. 15) en plus du célèbre dipinto de la felouque souvent évoquée (Basch 1993, p. 28 et fig. 24). Plusieurs de ces exemples, sont manifestement situés en contexte copte, notamment ceux provenant du monastère des Kellia situé à proximité d'Alexandrie. Or L. Basch a récemment montré que le terme «calfatage» qui se rapporte au sens strict au procédé de construction «sur membrure première» était d'origine copte et E. Rieth a mis en évidence qu'un des foyers d'origine parmi d'autres de cette construction était nilotique (Basch 2015; Rieth 2015). Dès lors, le rapprochement s'impose et si Alexandrie a été très certainement un centre important pour l'innovation des techniques nautiques, les Coptes ont manifestement joué un rôle dans leur développement, qu'il s'agisse de la construction «sur membrure première» ou de la voile latine ${ }^{35}$. Hypothèse qui rejoint celle de B. Kreutz qui voyait un certain parallélisme dans le développement de ces deux innovations, même si nous savons aujourd'hui que la transition technique de la construction sur bordé vers la construction sur membrure fut complexe comme le fut, sans doute aussi, celle de la voile latine ${ }^{36}$.

35. Sur le rôle des communautés monastiques coptes dans la batellerie égyptienne, cf. Fournet, Gascou 2002

36. Kreutz 1976. Sur la transition technique de la construction navale, cf. Pomey et al. 2012. 

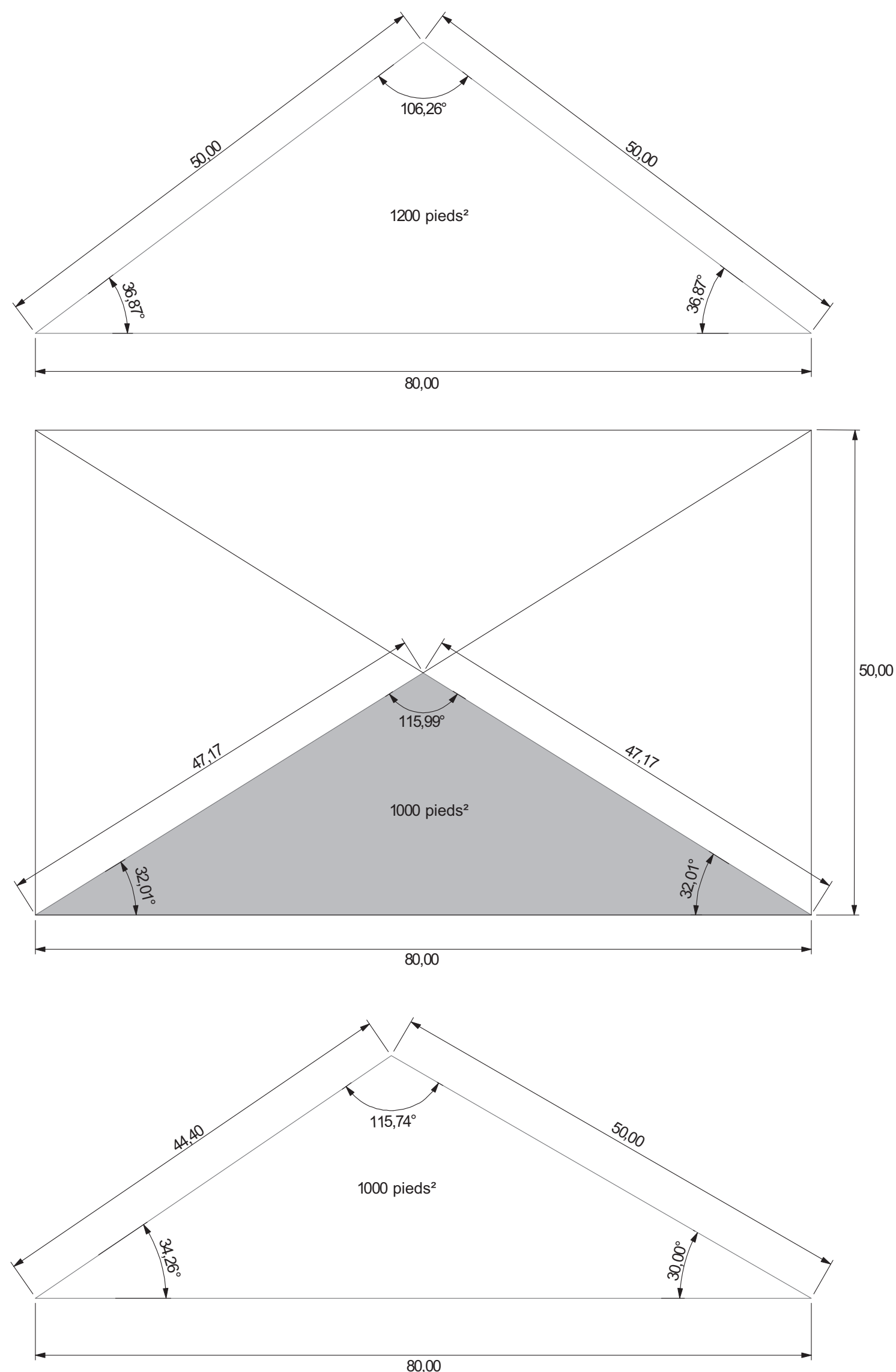

Fig. 14: Construction géométrique correspondant à la formule des Stereometrica (II, 48-49).1- Schéma du triangle correspondant à la voile aux dimensions données par Héron d'Alexandrie et à la surface calculée par la formule. 2- Schéma du rectangle construit sur les deux dimensions connues de la voile. Sa division par les diagonales permet d'obtenir deux triangles isocèles de même base et même surface que la voile d'origine mais dont les deux côtés égaux ont des dimensions approchées. 3- Schéma d'un voile en forme de triangle isocèle construit sur les dimensions données par Héron d'Alexandrie. La surface ne correspond pas au résultat attendu. Cette solution ne peut être retenue (Dessin P. Poveda Centre Camille Jullian). 


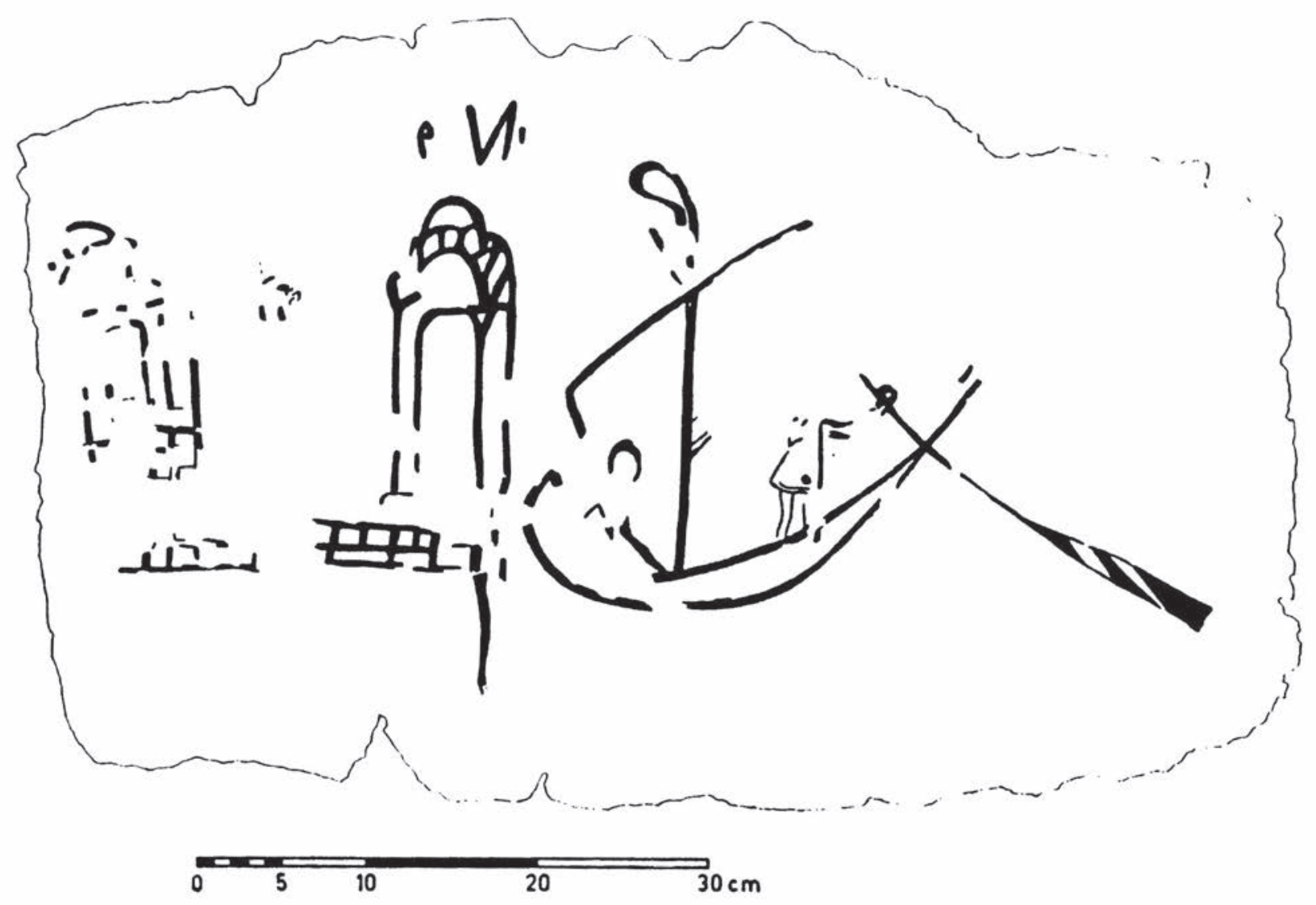

Fig. 15: Embarcation à voile latine provenant des Kellia (VII siècle) (Basch 1993).

\section{UN DOSSIER ENRICHI ET DE NOUVELLES INTERROGATIONS}

Au terme de cette étude, si le dossier de la voile latine reste ouvert, il s'est néanmoins enrichi de deux documents remarquables de nature à apporter un nouvel éclairage sur la question. Découverte il y a vingt-cinq ans, diffusée dans les milieux de l'archéologie navale depuis une dizaine d'années et souvent citée depuis, la mosaïque de Kelenderis n'avait jamais fait l'objet d'une étude approfondie jusqu'à ce jour. Il s'agit pourtant d'un document exceptionnel par son originalité et sa qualité artistique, mais aussi, du point de vue qui nous intéresse, par la précision des détails techniques concernant le gréement latin antique dans la version de la voile latine orientale. Par sa datation $\mathrm{au} \mathrm{V}^{\mathrm{e}}$ siècle, la mosaïque de Kelenderis reste à ce jour le document le plus ancien établi avec certitude attestant de l'usage de la voile latine en Méditerranée à la fin de l'Antiquité. Son degré d'évolution indique néanmoins une origine bien plus ancienne encore que de nombreux indices conduisent, peut-être dès le changement d'ère, à situer dans le contexte alexandrin. À cet égard, le texte des Stereometrica d'Héron d'Alexandrie, jusqu'à présent méconnu, constitue malgré sa datation et son origine incertaines un nouvel élément pouvant conforter cette hypothèse. En tout état de cause, il témoigne de l'importance prise par la voile latine dans la Méditerranée du premier millénaire, au point de devenir l'objet d'une formule pratique courante pour en mesurer la surface.

Mais si les questions qu'était amené à se poser P. Paris au milieu du siècle dernier (Paris 1949) sur cette voile «Voile latine? voile arabe? voile mystérieuse» apparaissent aujourd'hui largement dépassées, le navire de la mosaïque de Kelenderis n'en soulève pas moins de nouvelles questions. Ainsi après avoir connu un important développement, la disparition de la voile latine orientale accompagnée de ses bandes de ris reste pour le moins problématique. On le voit, bien qu'enrichi de nouveaux documents remarquables, le dossier de la voile latine, et en particulier de la voile latine orientale, continue de poser de nombreuses interrogations.

Patrice Pomey Directeur de recherche émérite. Aix Marseille Univ, CNRS, Minist Culture, CCJ, pomey@mmsh.univ-aix.fr 


\section{BIBLIOGRAPHIE}

ADAM P.

1970 À propos des origines de la voile latine, dans M. Cortelazzo, C. Villain-Gandossi (éd.), Méditerranée et Océan Indien, Travaux du Sixième Colloque International d'Histoire Maritime, Venise 1962, Paris, SEVPEN, p. 203-229.

BALTY J.

1977 Mosaïques antiques de Syrie, Bruxelles, Musées royaux d'art et d'histoire.

1995 Mosaïques antiques du Proche-Orient. Chronologies, iconographie, interprétation, Paris, Les Belles-Lettres (Annales littéraires de l'Université de Franche-Comté).

BASCH L.

1987 Le musée imaginaire de la marine antique, Athènes, Institut Hellénique pour la Préservation de la Tradition Nautique.

1989 The Way to the Lateen Sail, The Mariner's Mirror, 75, p. 328-332.

1991a Un navire marchand byzantin à Corinthe, Neptunia, 181, p. 14-21.

1991b La felouque des Kellia. Un navire de mer à voile latine en Égypte au $\mathrm{VII}^{\mathrm{e}}$ siècle de notre ère, Neptunia, 183, p. 2-10.

1993 Navires et bateaux coptes: état de la question en 1991, GraecoArabica, V, (Athènes), p. 23-62.

1997 L'apparition de la voile latine en Méditerranée, dans D. Garcia, D. Meeks (éd.), Techniques et économie antiques et médiévales. Le temps de l'innovation. Colloque d'Aix-en-Provence (mai 1996), Paris, Édition Errance, p. 214-223.

2001 La voile latine, son origine, son évolution et ses parentés arabes, dans H. Tzalas (ed.), Tropis VI, $6^{\text {th }}$ International Symposium on Ship Construction in Antiquity, Lamia 1996 proceedings, Athènes, Hellenic Institute for the Preservation of Nautical Tradition, p. 55-85. 2015 Le calfatage. L'origine du mot et son aire d'extension, dans P. Pomey (éd.), La batellerie égyptienne. Archéologie, histoire, ethnographie, Alexandrie, Centre d'Études Alexandrines (Études Alexandrines 34), p. 227-234.

BEAUdouin F.

1990 Bateaux des côtes de France, Paris, Éd. Glénat.

Boetto G.

2009 New Archaeological Evidence of the Horeia-Type Vessels: The Roman Napoli C Shipwreck from Naples (Italy) and the Boats of Toulon (France) compared, dans R. Bockius (ed.), Between the Seas. Transfer and Exchange in Nautical Technology. Proceedings of the Eleventh International Symposium on Boat and Ship Archaeology, Mainz 2006, Mayence, Römisch-Germanisches Zentralmuseum, p. 289-296.

2014 La batellerie portuaire, dans P. Pomey (dir), Ports et navires dans l'Antiquité et à l'époque byzantine, Dossiers d'Archéologie, 364, juillet/août, p. 56-61.

Boetto G., Poveda P.

2014 La restitution de Napoli C. Un navire roman à tableau, dans P. Pomey (dir.), Ports et navires dans l'Antiquité et à l'époque byzantine, Dossiers d'Archéologie, 364, juillet/août, p. 64-65.

BURLET R

1988 La voile latine, Neptunia, 171, p. 11-21.

Cadoret B., Maho Cl., Cadoret M.

2009 Le grand livre des voiles \& gréements, Grenoble, Éd. Chasse-Marée, Glénat.

CASSON L.

1966 The Lateen Sail in the Ancient World, The Mariner's Mirror, 52, p. 199-200.

1971 Ships and Seamanship in the Ancient World, Princeton University Press, Princeton.

2006 Which End is Which? IJNA, 35.2, p. 331.

Donceel-Voute P.

1988 Les pavements des églises byzantines de la Syrie et du Liban-décor, archéologie et liturgie, Louvain-la-Neuve, Département d'archéologie et d'histoire de l'art, Collège Erasme.
1994 Le ve siècle dans les mosaïques de Syrie, dans J.-P. Darmon et

A. Rebourg (éd.), La mosaïque gréco-romaine IV, Actes du

IV Colloque de l'AIEMA, Trèves, 1984, Paris, AIEMA, p. 205-210.

\section{Duval P.-M.}

1949 La forme des navires romains d'après la mosaïque d'Althiburus, $M E F R, 61$, p. 119-149.

Fournet J.-L., GASCOU J.

2002 Moines pachômiens et batellerie, dans Ch. Décobert (éd.), Alexandrie médiévale 2, (Études Alexandrines 8), IFAO, Le Caire, p. 23-45.

FriEdMAN Z.

2003 Ship Iconography in Mosaics-An Aid to Understanding Ancient Ships and Their Construction. PhD thesis, Department of Maritime Civilizations, University of Haifa, (non publiée).

2007 Reply: the Kelenderis Ship, IJNA, 36.2, p. 417-419.

Friedman Z., Zoroglu L.

2006 Kelenderis Ship - Square or Lateen Sail?, IJNA, 35.1, p. 108-116.

Gassend J.-M., Liou B., Ximénès S.

1984 L'épave 2 de l'Anse des Laurons (Martigues, Bouches-du-Rhône), Archaeonautica, 4, p. 75-105.

\section{Gates M.-H.}

1994 Archaeology in Turkey, AJA, 98, 2, p. 277-278.

1995 Archaeology in Turkey, AJA, 99, 2, p. 248-249.

Heiberg J. L.

1976 Stereometrica (Heronis Alexandrini Opera quae supersunt omnia), vol. 5, Leipzig, Edition Teubner, 1914 (réed. 1976).

Huet J., Rigaud Ph., Vigne B.

2004 Voiles latines. Renaissance des bateaux de Méditerranée, Douarnenez, Éd. du Chasse-Marée (Bateaux des côtes de France, 1).

KREUtZ B.M.

1976 Ships, Shipping and the Implication of Change in the Eary Medieval Mediterranean, Viator, 7, p. 79-109.

MAIURI A.

1958 Navalia Pompeiana, Rendiconti dell'Accademia di Archeologia, Lettere e Belli Arti, Naples, 33, p. 7-34.

PARIS P.

1949 Voile latine ? Voile arabe ? Voile mystérieuse, Hespéris, 36, p. 69-96.

Polzer M. E

2008 Toggles and Sails in the Ancient World: Rigging Elements Recovered from the Tantura B Shipwreck, Israel, IJNA, 37.2, p. 225252.

Pomey P.

1993 Le navire de Cucuron: un graffito décoratif, Archaeonautica, 11, p. $149-163$

1997a L'art de la navigation dans l'Antiquité, Actes du Colloque Regards sur la Méditerranée, Paris, Académie des Inscriptions et BellesLettres (Cahiers de la Villa Kérylos, 7), p. 89-101.

1997b P. Pomey (dir.), La Navigation dans l'Antiquité, Aix-en-Provence, Édisud.

2006 The Kelenderis Ship: A Lateen Sail, IJNA, 35.2, p. 326-329.

2011 A Comparative Study of Sewn Boats from the Mediterranean, the Red Sea and the Indian Ocean. The question of Gujarat, dans L. Varadarajan, Gujarat and the Sea, Vadodara, Gujarat (India), p. 133-146.

Pomey P., Kahanov Y., Rieth E.

2012 Transition from Shell to Skeleton in Ancient Mediterranean ShipConstruction: analysis, problems, and future research", IJNA, 41, 2, p. 235-314. 
Rieth E.

Pour une approche nilotique des origines ( $\mathrm{V}^{\mathrm{e}}-\mathrm{VII}^{\mathrm{e}}$ siècle) de la construction navale «sur membrure première» en Méditerranée, dans P. Pomey (éd.), La batellerie égyptienne. Archéologie, histoire, ethnographie, Alexandrie, Centre d'Études Alexandrines (Études Alexandrines 34), p. 201-225.

2016 Méditerranée et Océan Indien : histoires parallèles, dans Aventuriers des mers. VII ${ }^{e}$-XVII . De Sindbad à Marco Polo, Paris, Marseille, Vanves, IMA/MUCEM.

RouGÉ J.

1978 Romans grecs et navigation: le voyage de Leucippé et Clitophon de Beyrouth en Égypte, Archaeonautica, 2, p. 265-280.

ROBERTS O. T. P.

2006 The Rig of the Kelenderis Ship Reconsidered, IJNA, 35.2, p. 330331.

VIGNE B.

1994

Les derniers pointus de Raphaël Autiéro, Le Chasse-Marée, 79, p. 14-27.

VITRAC B

2010 Héron d'Alexandrie et le corpus métrologique: état des lieux, dans Géométrie(s), pratiques d'arpentage et enseignement: quels liens et dans

$$
\text { quel contexte?, Paris, }
$$
0047398).

\section{WhiteWRIGHT J.}

2009 The Mediterranean Lateen Sail in Late Antiquity, IJNA, 38.1, p. 97-103.

2011 Efficiency or economics? Sail development in the Ancient Mediterranean", dans W. V. Harris, K. Iara (ed.), Maritime Technology in the Ancient Economy: Ship-Design and Navigation, Portsmouth, Rhode Island (JRA, Suppl. 84) p. 89-102.

\section{ZOROGLU L.}

1992 Kelenderis 1992, Yili Kelenderis Kazi ve Onarim Çalismalari, XV. Kazi Sonuçlari Toplantisi II, Ankara 1993, p. 189-209.

1993 Bir Mozaik Üzerinde Kelenderis Betimlemesi, 1993 Yili Anadolou Medeniyetleri Müzesi Konferanslari, Ankara 1994, p. 31-40

1994 Kelenderis 1994, Yili Kelenderis Kazi ve Onarim Çalismalari, XVII. Kazi Sonuçlari Toplantisi II, Ankara 1995, p. 263-276.

1996 Kelenderis Mozaigi, Çaglar Boyunca Anadolu'da Yerlesim ve Konut Uluslararasi Sempozyumu, 5-7 Haziram 1996, (International Symposium on Settlement and Housing in Anatolia Through the Ages, 5-7 June 1996), p. 514-524. 